\title{
Altered Redox Homeostasis in Branched-Chain Amino Acid Disorders, Organic Acidurias, and Homocystinuria
}

\author{
Eva Richard, Lorena Gallego-Villar, Ana Rivera-Barahona, Alfonso Oyarzábal, Belén Pérez, \\ Pilar Rodríguez-Pombo, and Lourdes R. Desviat
}

Centro de Biología Molecular Severo Ochoa UAM-CSIC, Centro de Diagnóstico de Enfermedades Moleculares (CEDEM), CIBERER, IdiPaz, Universidad Autónoma de Madrid, Madrid, Spain

Correspondence should be addressed to Lourdes R. Desviat; lruiz@cbm.csic.es

Received 29 September 2017; Revised 26 December 2017; Accepted 16 January 2018; Published 20 March 2018

Academic Editor: Maria C. Franco

Copyright (C) 2018 Eva Richard et al. This is an open access article distributed under the Creative Commons Attribution License, which permits unrestricted use, distribution, and reproduction in any medium, provided the original work is properly cited.

\begin{abstract}
Inborn errors of metabolism (IEMs) are a group of monogenic disorders characterized by dysregulation of the metabolic networks that underlie development and homeostasis. Emerging evidence points to oxidative stress and mitochondrial dysfunction as major contributors to the multiorgan alterations observed in several IEMs. The accumulation of toxic metabolites in organic acidurias, respiratory chain, and fatty acid oxidation disorders inhibits mitochondrial enzymes and processes resulting in elevated levels of reactive oxygen species (ROS). In other IEMs, as in homocystinuria, different sources of ROS have been proposed. In patients' samples, as well as in cellular and animal models, several studies have identified significant increases in ROS levels along with decreases in antioxidant defences, correlating with oxidative damage to proteins, lipids, and DNA. Elevated ROS disturb redoxsignaling pathways regulating biological processes such as cell growth, differentiation, or cell death; however, there are few studies investigating these processes in IEMs. In this review, we describe the published data on mitochondrial dysfunction, oxidative stress, and impaired redox signaling in branched-chain amino acid disorders, other organic acidurias, and homocystinuria, along with recent studies exploring the efficiency of antioxidants and mitochondria-targeted therapies as therapeutic compounds in these diseases.
\end{abstract}

\section{Introduction}

A growing body of evidence indicates that disruption of redox homeostasis is involved in the pathophysiology of several inborn errors of metabolism (IEMs), including organic acidurias, respiratory chain disorders, fatty acid oxidation disorders, and aminoacidopathies [1-7]. IEMs are defined as genetic defects that affect a metabolic pathway or cellular process and are commonly characterized by a specifically altered biochemical profile that guides the diagnosis [8]. They belong to the category of rare diseases (defined by the EU as those with an incidence lower than 1 in 2000), and most of them are inherited in autosomal recessive fashion. In the classic IEMs, pathology results from accumulation of toxic metabolites and/or from the deficiency or absence of substrates necessary to perform normal cellular functions. Many of them are systemic, and in most cases, they affect the central nervous system [8].

Several IEMs share common pathomechanisms as a consequence of the accumulation of toxic metabolites; these inhibit specific mitochondrial enzymes and processes, resulting in mitochondrial dysfunction, impaired energy metabolism, increased reactive oxygen species (ROS) levels, altered antioxidant response capability, and increased oxidative stress, resulting in damage to DNA, proteins, and lipids $[2,3,9,10]$. The brain and heart, frequently affected in IEMs, are energy-demanding organs highly sensitive to oxidative stress due to their high rate of oxygen consumption, substantial iron and polyunsaturated lipid contents, and relatively low activity of their antioxidant defences and repair enzymes 
[11]. Metabolic profiling of oxidative stress in IEM patients' samples has documented the correlation between oxidative stress markers and biochemical and clinical parameters [12, 13].

Mitochondria, which are the major energy producers in the cell, and where many important metabolic pathways take place, are the major ROS producers [14]. Mitochondrial ROS is generated, in the form of superoxide anion $\left(\mathrm{O}_{2}{ }^{-}\right)$, at complexes I and III of the electron transport chain and are rapidly converted to hydrogen peroxide $\left(\mathrm{H}_{2} \mathrm{O}_{2}\right)$ by the enzyme superoxide dismutase (MnSOD). Mitochondrial matrix complexes such as pyruvate dehydrogenase, alpha-ketoglutarate dehydrogenase, or branched-chain alpha-ketoacid dehydrogenase $(\mathrm{BCKDH})$ can also generate $\mathrm{O}_{2}{ }^{-}$. Other cellular sources of ROS exist, and an active interplay between all of them has been documented [15]. To avoid the noxious consequences of ROS, mitochondria express a variety of antioxidant enzymes, including MnSOD, peroxiredoxins, and glutathione peroxidase (GPX) [16]. Among the nonprotein antioxidants, glutathione (L- $\gamma$-glutamyl-L-cysteinylglycine) represents the main antioxidant defence present in the cell. An increase in ROS levels correlates with a decrease in reduced glutathione (GSH). Thus, the ratio of oxidized (GSSG) to reduced (GSH) is indicative of cellular redox balance and has been used as a biomarker of oxidative stress in different diseases including IEMs [17-20].

At physiological concentrations, ROS are recognized to act as signaling molecules modifying specific proteins that will transmit a message modulating specific cellular pathways, in what is known as redox signaling [15]. Wellknown examples include redox-sensitive changes in conformation and function of receptors, transcription factors, kinases, and phosphatases, which lead to a specific cellular response [15]. At high concentrations, ROS may cause extensive damage to cells, by peroxidation of lipids, carbonylation of proteins, or DNA base oxidation. These toxic effects can disrupt mitochondrial function causing a further increase in ROS production, thus forming a vicious cycle. In cases of chronic, high levels of ROS production, apoptosis or autophagy/mitophagy processes are triggered [16]. All this has led to redefine the concept of oxidative stress, initially conceived as an alteration in the balance between oxidant and antioxidant molecules, to a disturbance in redox signaling with possible molecular and/or cellular damage [15].

In the mitochondria, $\mathrm{H}_{2} \mathrm{O}_{2}$ is the most likely signal involved in redox signaling and in transcription regulation, through thiol/disulfide exchange reactions [21]. Signal transduction by $\mathrm{H}_{2} \mathrm{O}_{2}$ may occur via peroxiredoxin- 2 and transcription factor STAT3 [22]. $\mathrm{H}_{2} \mathrm{O}_{2}$ acts on redoxsensitive targets such as transcription factor NRF2 involved in antioxidant response, $\mathrm{NF} \kappa \mathrm{B}$ protein complex involved in inflammation, stress kinase JNK, and insulin-like growth factor IGF1, linking energy metabolism and inflammatory responses [23]. Oxidative stress and bioenergetics deficits trigger signaling through mitophagy receptors such as ATG32, BCL2-L-13, and FUNDC1, leading to the elimination of damaged mitochondria [24].

In IEMs, as in cardiovascular and neurodegenerative diseases, the production of pathological amounts of ROS resulting in oxidative damage may indeed disturb redox signaling and induce cell stress responses, which may in some cases lead to cell death $[1,25]$. However, the exact molecular mechanisms and cellular pathways involved in this progression are scarcely characterized. In some IEMs, specific activation of stress kinases p38 and JNK and of autophagy/mitophagy and apoptotic pathways has been documented [6, 7, 26, 27].

A recent review provided insight into the adaptive stress responses that operate in IEMs, linking mitochondrial biogenesis to cellular antioxidant, anti-inflammatory, protein quality control, and autophagy functions [1]. As a first line of cellular responses to oxidative stress, the expression of antioxidant enzymes and heat-shock proteins increases, along with an upregulation of glycolysis and activation of mitochondrial biogenesis, as occurs in multiple acyl-CoA dehydrogenation deficiency [28]. ROS-mediated activation of AMP-activated protein kinase (AMPK) could be the link between metabolic reprogramming, mitochondrial biogenesis, and activation of cellular stress repair pathways, followed by the progression to proinflammatory responses controlled by signaling pathways mTOR/HIF- $1 \alpha / \mathrm{NF} \kappa B[1]$.

The presence of interconnected redox-signaling pathways and their alterations in disease contributing to the pathogenesis suggests that specific compounds targeting excessive ROS or mitochondrial dysfunction may show therapeutic potential. This has been explored in some IEMs, in which several antioxidants or mitochondrial biogenesis activators have proven effective in cellular and/or animal models of disease [29-32]. However, clinical efficacy of these approaches remains unproven. In addition, the possible interference of these therapies with cellular redox signaling should be taken into account for careful design and appropriate clinical testing.

In this study, we describe sources of ROS in specific IEMs; we summarize the available data on altered redox homeostasis and oxidative damage in patients' samples and in animal models of disease; we discuss potential implications for redox signaling and describe the recent advances in antioxidant- and mitochondria-targeted therapies for these pathologies. To this aim, we have focused in selected branched-chain amino acid disorders, organic acidurias, and defects leading to the accumulation of homocysteine (homocystinuria) (Figure 1). For other IEMs, namely inherited mitochondrial diseases and fatty acid oxidation disorders, the readers are referred to recent reviews $[9,33]$.

\section{Branched-Chain Amino Acid Disorders and Organic Acidurias}

Branched-chain amino acids (BCAAs: Val, Ile, Leu) are essential amino acids not only necessary for protein synthesis but also key nitrogen donors involved in interorgan and intercellular nitrogen shuttling [34] and key nutrient signals involved in important functions including food intake regulation. Leu has long been known as an anabolic nutrientsignaling molecule that stimulates protein synthesis by activation of the mTORC1 pathway in selected tissues [35]. Recently, mitochondrial sirtuin 4 (SIRT4), acting as a lysine 


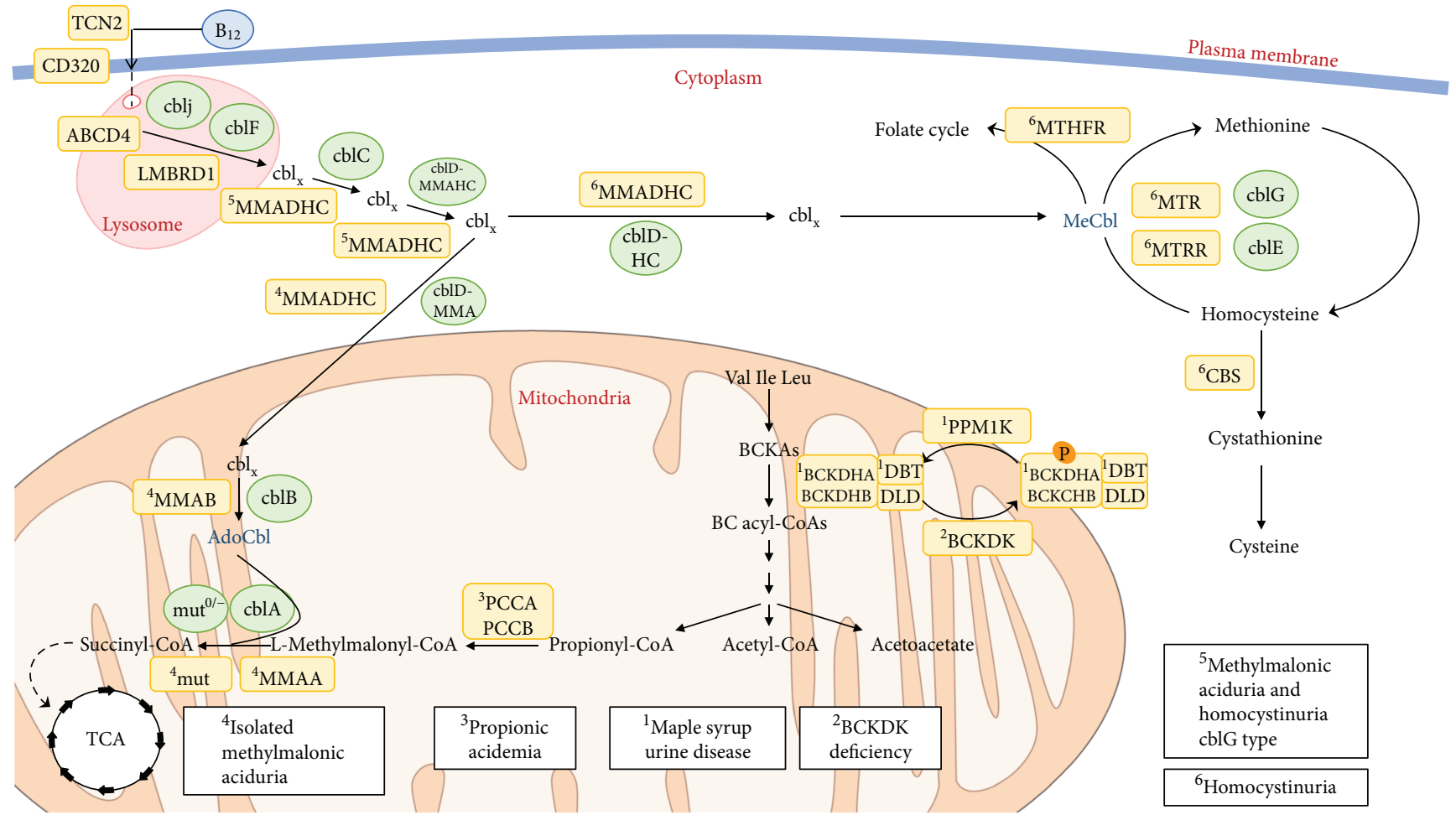

FIGURE 1: Schematics of the metabolic pathways and their cellular localization affected in branched-chain organic acidurias and homocystinuria disorders mentioned in this work. The affected genes and corresponding diseases (in boxes) are shown for each pathway.

deacylase, has been reported to control Leu catabolism and insulin secretion, reinforcing the hypothesis of a possible link between BCAA metabolism and insulin secretion [36]. All these data underscore the critical role of BCAAs metabolism in humans' health and disease, as was evidenced with the characterization of maple syrup urine disease (MSUD) [37] and, more recently, with the description of branched-chain ketoacid dehydrogenase kinase (BCKDK) deficiency $[38,39]$.

Organic acidurias/acidemias are described as IEMs affecting enzymes, receptors, or transport proteins in the catabolic pathway of amino acids and odd-chain fatty acids and characterized by the abnormal accumulation of organic acids in body fluids [40]. More than 65 organic acidurias have been described, with MSUD, propionic acidemia (PA), and methylmalonic acidurias (MMA) among the most prevalent. A secondary mitochondrial dysfunction induced by accumulating toxic metabolites is an important pathomechanism in these disorders [10]. Patients with organic acidurias show elevated plasma protein carbonyls and lower GSH levels in leukocytes [20]. Here we briefly summarize the published data on branched-chain amino acid disorders and specific organic acidurias with a profile of altered redox homeostasis.

\subsection{Disorders Involving Branched-Chain $\alpha$-Ketoacid} Dehydrogenase. MSUD (MIM \#248600), caused by the deficiency of branched-chain $\alpha$-ketoacid dehydrogenase complex (BCKDHc) activity, is characterized by elevated levels of BCAAs and their corresponding $\alpha$-keto-acids (BCKAs) in body fluids and tissues, resulting in complex neurological phenotypes [37]. As the important gatekeeping enzyme that it is, $\mathrm{BCKDHc}$ is regulated by reversible phosphorylation catalyzed by a specific BCKD kinase (BCKDK) that inhibits BCKDHc function, halting the catabolic pathway of BCAAs [41], and a dephosphorylation catalyzed by the mitochondrial protein phosphatase $\mathrm{PP} 2 \mathrm{Cm}$ (encoded by the PPM1K gene) that stimulates $\mathrm{BCKDHc}$ activity [42]. Optimal $\mathrm{BCKDHc}$ activity necessary to maintain BCAA homeostasis is achieved by the coordinated response of BCKDK and PP2Cm activities. Blockage or unrestrained BCAA metabolism through BCKDHc leads to a dysmetabolism of BCAAs resulting in MSUD or BCKDK deficiency (MIM \#614923) (Figure 1), two different clinical conditions with a hallmark of neurological perturbation.

MSUD results from mutations in the genes E1 $\alpha$ BCKDHA (MIM \#608348), E1 $\beta-B C K D H B$ (MIM \#24861), and E2-DBT (MIM \#248610) [37]. The disease affects $1: 185,000$ newborns worldwide and is manifested by diverse clinical phenotypes, ranging from the most severe form-seen in 70\% of patients with MSUD and associated with a profound neurological impact and high mortality if not treated early-to mild forms that present during early development. The mechanisms underlying brain injury are not completely understood. Different studies have been carried out using chemical induction of the disease by BCAAs or BCKAs in cultured cells $[43,44]$ and animal models [45-48]. All converge in the identification of oxidative stress, brain energy deficit, and/or alterations in the brain's neurotransmission balance, mostly affecting 
glutamate [46], as important neurodegenerative determinants. Recent studies in human peripheral blood mononuclear cells have shown that $10 \mathrm{mM}$ of BCAAs can directly trigger a mechanism that involves the two major ROS sources, NADPH oxidase and mitochondria. BCAAs stimulate the activation of the redox-sensitive transcription factor $N F \kappa B$ resulting in the release of proinflammatory molecules [49].

Increases in lipid and protein oxidation are detected in plasma of MSUD patients [50, 51]. The two branchedchain aminotransferases (BCATs) involved in the conversion of BCAAs in BCKAs contain redox-active cysteine residues and could be affected by an oxidative environment. Their inhibition would provoke a further increase in the BCAA levels that could exacerbate the effect of the blockage [34]. An increased inflammatory profile has also been observed in plasma samples from MSUD patients maintained at low BCAA levels indicating the presence of sustained inflammation and activation of the immune system probably as a result of unbalanced ROS production [52].

On the other hand, mutations in the PPM1K gene in human result in a mild increase of BCAA and BCKA levels compatible with a mild variant form of MSUD (Figure 1), which nevertheless results in ROS increases along with the activation of the JNK and p38 MAP kinases [42]. This could be related to its critical role for mitochondrial function and cell survival previously described in animal models [53].

In the reverse of the MSUD metabolic problem, BCKDK patients (MIM \#614923) manifest an unrestrained BCAA metabolism with decreased BCAA and BCKA levels, accompanied by neurocognitive phenotypes $[38,39]$. However, also in this case, the mitochondrial response to BCKDK deficiency seems to be the increase in $\mathrm{O}_{2}{ }^{-}$, alterations in the expression of MnSOD and GPX, and alterations in the bioenergetics' profile with reductions in ATP-linked respiration and intracellular levels. All these data support the hyperfusion response of $\mathrm{BCKDK}$-deficient mitochondria to stress and the altered cell fate observed in patients' fibroblasts [54]. Apart from the canonical ROS-producing sites in mitochondrial electron transfer chain, several reports pointed towards the important role of mitochondrial 2-oxoacid dehydrogenase complexes (2-oxoglutarate dehydrogenase, pyruvate dehydrogenase, and $\mathrm{BCKDH}$ ) that share the E3 dihydrolipoamide dehydrogenase, as major $\mathrm{O}_{2}{ }^{-} / \mathrm{H}_{2} \mathrm{O}_{2}$ producers under conditions of maximum activities of substrate oxidation $[55,56]$. Therefore, the most likely result from the uninhibited activity of BCKDHc could be a misbalance in redox equilibrium and an increase in the production of $\mathrm{O}_{2}{ }^{-} / \mathrm{H}_{2} \mathrm{O}_{2}$ [54-56].

2.2. Propionic Acidemia. Propionic acidemia (PA, MIM \#606054) is one of the most frequent life-threatening organic acidurias, affecting 1 in 100,000 live births worldwide. PA results from mutations in the $P C C A$ or $P C C B$ gene that encode the $\alpha$ and $\beta$ subunits of propionyl-CoA carboxylase (PCC), respectively. PCC is a biotin-dependent mitochondrial enzyme that catalyzes the reaction of propionyl-CoA to D-methylmalonyl-CoA, the first step of the propionate oxidation pathway (Figure 1). Propionyl-CoA derives from the catabolism of certain amino acids including BCAAs (Ile, Val, Thr, and Met), of cholesterol, and from the betaoxidation of odd chain fatty acids and bacteria gut production [57]. PCC deficiency results in the accumulation and excretion of propionate, 3-hydroxypropionate, methylcitrate, and propionylglycine, which are the biochemical hallmarks for diagnosis [57]. PA leads to a multisystemic disorder that affects the cardiovascular, gastrointestinal, renal, nervous, and immune systems $[58,59]$. The clinical outcome varies from a severe neonatal form characterized by ketoacidosis, feeding refusal, lethargy, failure to thrive, seizures, and encephalopathy to a milder-late onset form with metabolic decompensations, failure to thrive, and diverse neurological deficiencies [60-62]. Cardiomyopathy and neurological features are associated with a high morbidity and mortality and have been attributed to bioenergetic failure and intracellular accumulation of toxic metabolites [63, 64].

A number of in vitro and in vivo studies of propionylCoA metabolites have shown inhibition of enzymes involved in energy production pathways, such as respiratory chain complex III [65] and pyruvate dehydrogenase complex [66]. Furthermore, propionyl-CoA reacts with oxaloacetate to produce methylcitrate that inhibits enzymes such as phosphofructokinase aconitase and citrate synthase [67]. In addition, propionate, at concentrations similar to those found in the plasma of PA patients, strongly inhibits oxygen consumption as well as oxidation of pyruvate and alphaketoglutarate in rat liver mitochondria $[66,68]$. Moreover, the lack of PCC that blocks the anaplerotic biosynthesis of succinyl-CoA from propionyl-CoA may result in diminished TCA cycle activity [69]. This could be especially relevant for the heart and skeletal muscle, for which propionate is a major anaplerotic substrate. Propionic acid was shown to stimulate the production of $\mathrm{O}_{2}{ }^{-}$in the presence of $\mathrm{Ca}^{2+}$ influx activators in human neutrophils [70], to increase protein carbonylation in rats [71] and to stimulate lipid peroxidation in rat cerebral tissues [72].

The secondary mitochondrial dysfunction in PA is manifested as a decrease in ATP and phospho-creatine production, a decrease in the activity of respiratory chain complexes, mtDNA depletion, and abnormal mitochondrial structures present in PA patients' biopsies. This is evident particularly in high-energy-demanding organs such as the brain and heart [73-75]. In addition, evidence of oxidative stress and cellular damage has been shown in PA patients' fibroblasts through detection of elevated intracellular $\mathrm{H}_{2} \mathrm{O}_{2}$ levels correlating with the activation of the JNK and p38 pathways [26]. Urinary samples from PA patients show high levels of oxidative stress markers [12].

Alterations in redox homeostasis and mitochondrial function were observed in a hypomorphic mouse model of PA $\left(\mathrm{Pcca}^{-1-}(\mathrm{A} 138 \mathrm{~T})\right)$ [5]. These include increased $\mathrm{O}_{2}{ }^{-}$production and in vivo mitochondrial $\mathrm{H}_{2} \mathrm{O}_{2}$ levels, mtDNA depletion, lipid oxidative damage, and tissue-specific alterations in the activities of OXPHOS complexes and in antioxidant defences. An increase in the DNA repair enzyme 8guanine DNA glycosylase 1 (OGG1) induced by oxidative stress was also found in the liver of PA mice [32]. These 
alterations show a good correlation with the altered mitochondrial function and oxidative damage detected in PA patients' samples [12, 73-77].

2.3. Methylmalonic Acidurias. Methylmalonic acidurias (MMA) include a heterogeneous group of autosomal recessive genetic disorders caused by deficiency of the mitochondrial enzyme methylmalonyl-CoA mutase (MCM) or by defects in cobalamin (cbl) absorption, intracellular cbl trafficking, or synthesis of adenosylcobalamin (Adocbl) and methylcobalamin (Mecbl), which serve as cofactors for MCM and for the cytosolic enzyme methionine synthase, respectively (Figure 1). Two clinical entities have been described: (i) isolated MMA, characterized by methylmalonic acid accumulation and caused by MCM deficiency, defects in Adocbl synthesis, and methylmalonyl-CoA epimerase deficiency and (ii) combined MMA and homocystinuria, characterized by methylmalonic acid and homocysteine accumulation and caused by defects in intracellular cbl metabolism (Figure 1).

2.3.1. Isolated MMA. MCM (MIM \#251000; $m u t^{0}$ or $m u t^{-}$ enzymatic subtypes), encoded by the mut gene, is a mitochondrial matrix enzyme that catalyzes the conversion of L-methylmalonyl-CoA to succinyl-CoA [57]. Biochemically, the disease is characterized by accumulation of methylmalonic acid and by accumulation of propionate, 3-hydroxypropionate, and 2-methylcitrate, due to activation of alternative pathways of propionate oxidation. Clinical presentation varies from a severe, early onset form of the disease, with neonatal ketoacidosis, lethargy, failure to thrive, encephalopathy, and hepatomegaly, to a milder late-onset presentation usually diagnosed during infancy that has a less serious neurological outcome [57]. Neurological symptoms, including psychomotor delay, irritability, lethargy, hypotonia, convulsions, and coma, have been related to a failure in mitochondrial oxidative metabolism. Mitochondrial dysfunction could be due to a combination of the inhibition of specific enzymes and transporters, limitation in the availability of substrates for mitochondrial metabolic pathways, and oxidative damage [78].

Mitochondrial toxicity of methylmalonic acid has been examined in vitro using a variety of normal tissues and extracts, including rat brain, mouse muscle, and bovine heart, suggesting an impairment of mitochondrial function, ROS generation, and other secondary metabolic perturbations [72, 79-81]. In addition, 2-methylcitric acid, malonic acid, and propionyl-CoA inhibit respiratory chain and tricarboxylic cycle [82]. In cultured neurons, methylmalonic acid decreases ATP/ADP ratio, collapses ion gradients, causes membrane depolarization, and increases intracellular calcium levels, leading to necrotic and apoptotic cell death [78].

Experimental evidence using patients' samples and a mut-knockout mouse identified multiple oxidative phosphorylation (OXPHOS) defects in MMA perturbations in antioxidant defences and resulting oxidative damage, indicating deficient energy metabolism caused by methylmalonyl-CoA accumulation and reduced succinylCoA levels [73, 83-86]. Proteomic analysis of patient liver samples by two-dimensional differential in-gel electrophoresis (2D-DIGE) and mass spectrometry (MALDI-MS and LCMSMS) showed alterations of enzymes involved in free radical scavenging [87]. In patient's fibroblasts, proteomic analysis (2-D DIGE/MALDI-TOF) identified several differentially expressed proteins related to oxidative stress and apoptosis (cytochrome c, MnSOD, and mitochondrial glycerophosphate dehydrogenase) [88]. In a study using high-dimensional flow cytometry, patients with MMA showed lower GSH levels in T lymphocytes, monocytes, and neutrophils, compared to healthy controls [20]. Oxidative damage markers (F-2 isoprostanes and dityrosine) were found increased in urinary samples from MMA patients [12, 89]. Plasma from patients showed significantly higher levels of protein carbonyls as compared to healthy controls [20].

In addition, various parameters of oxidative stress and apoptosis were evaluated in cultured fibroblasts from a spectrum of patients with MMA showing a significant increase in intracellular ROS content, MnSOD protein and apoptosis rate compared to controls [6]. Furthermore, cytochrome c oxidase deficiency was observed in renal tubular megamitochondria from a MMA patient [90], and mitochondrial ultrastructural abnormalities were found in renal samples from MMA mice [89] and in liver samples from MMA $m u t^{0}$ patients undergoing transplantation [91].

2.3.2. MMA Combined with Homocystinuria. Cobalamin deficiency type $\mathrm{C}$ with methylmalonic aciduria and homocystinuria (cblC; MMACHC; MIM \#277400 and \#609831) (Figure 1) is the most frequent genetic disorder of vitamin $\mathrm{B}_{12}$ metabolism. MMACHC protein is a processing enzyme involved in an early cytoplasmic step in the cofactortrafficking pathway. It exhibits unusual chemical versatility with demonstrated gluthatione transferase, reductive decyanase, and aquocobalamin reductase activities and converts incoming cobalamin derivatives to an intermediate that can be partitioned into Mecbl and Adocbl synthesis to support cellular needs [92-94]. MMACHC catalyzes the glutathione(GSH-) dependent dealkylation of alkylcobalamins and the reductive deacyanation of cyanocobalamin [95].

Early-onset patients with cblC disease present multisystem failure while there are late-onset patients exhibiting a milder form, with progressive neurological symptoms and behavioural disturbances [96]. Maculopathy and abnormal vision are extremely common in early-onset patients [97].

Increasing evidence provides support that oxidative stress is closely associated with the physiopathology of this disorder. In this disease, there is a combined contribution of mitochondrial ROS due to accumulation of methylmalonic acid, as well as of homocysteine-derived ROS (see below, the Homocystinuria section). Patient-derived fibroblasts showed elevated ROS, apoptosis, and active phosphorylated forms of p38 and JNK stress-kinase levels [98, 99]. High levels of oxidative damage biomarkers have been found in urinary samples [12]. Decreased levels of proteins involved in cellular detoxification (members of the peroxiredoxin family and isoforms of glutathione-S-transferases) were detected in patient-derived fibroblasts by proteomics techniques $[100,101]$. Another proteomic study in patients' 
lymphocytes revealed the deregulation in the expression of proteins involved in oxidative stress and cellular detoxification, especially those related to glutathione metabolism [102]. In relation to this, an imbalance of glutathione metabolism was observed in $c b l C$ blood samples with a significant decrease in total and reduced glutathione, along with an increase in different oxidized glutathione forms [19]. GSH depletion could be the consequence of a reduced synthesis since total glutathione and its rate-limiting precursor (cysteine) is decreased in $c b l C$ patients [19]. In addition, increased levels of protein-bound glutathione were detected, providing evidence of the impact of changes in glutathione pools in the pathogenesis of $c b l C$ disease through redox regulation of protein function [19]. Furthermore, two mutant MMACHC proteins exhibited impaired glutathione binding, and stabilization of the cob(II)alamin derivative has been observed in presence of physiologically relevant glutathione concentrations. The preferential stabilization of cob(II)alamin by the mutants with a concomitant increase in GSSG production offers insights into ROS production [103].

2.4. Glutaric Aciduria Type I. Glutaric aciduria type I (GAI, OMIM \#231670) is caused by the deficiency of the mitochondrial enzyme glutaryl-CoA dehydrogenase (GCDH), responsible for the oxidative decarboxylation of glutarylCoA to crotonyl-CoA, in the catabolic pathways of lysine and tryptophan [104]. The deficiency causes accumulation of glutarate and 3-hydroxyglutarate, and patients are at risk of acute striatal injury during encephalopathic crises before 4 years of age, frequently precipitated by nonspecific illnesses [105]. These episodes lead to the appearance of neurological symptoms including dystonia, dyskinesia, seizures, and coma [106]. Current treatment for GAI includes dietary lysine restriction and carnitine supplementation, although this does not prevent striatal degeneration in about one-third of the patients. Excitotoxicity, oxidative stress, and mitochondrial dysfunction induced by accumulating metabolites have been associated with brain pathogenesis, although the exact underlying mechanisms remain unclear $[4,107,108]$.

Glutaric acid administration induces lipid peroxidation and alterations in antioxidant defences in rats [109]. GCDH-deficient knockout mice $\left(G c d h^{-1-}\right)$ show a biochemical phenotype comparable to GAI patients but do not develop striatal degeneration [110]. These mice exhibit protein oxidative damage and reduction of antioxidant defences in the brain when subjected to an acute lysine overload [111]. Indeed, GCDH knockout mice fed on a high-lysine chow represent an animal model of GAI encephalopathy $g$ [112]. In these mice, high concentrations of glutaric acid within neurons were correlated with mitochodrial swelling and biochemical changes (depletion of alpha-ketoglutarate and accumulation of acetyl-CoA) consistent with Krebs cycle disruption [113].

2.5. Other Organic Acidurias. There are several other organic acidurias in which there is an inhibition of energy metabolism attributed to accumulation of toxic metabolites in mitochondria. These include 3-methylcrotonyl-CoA carboxylase deficiency (MCC, MIM \#210200 and \#210210), 2-methyl-3-hydroxybutyric aciduria (MOHBA, MIM \#20 3750), 3-methylglutaconic aciduria (MHGA, MIM \#250 950), D-2-hydroxyglutaric aciduria (D-2-OHGA, MIM \#600721), L-2-hydroxyglutaric aciduria (L-2-OHGA, MIM \#236792), 3-hydroxy-3-methylglutaric aciduria (3-HMGA, MIM \#246450), and ethylmalonic encephalopathy (EE, MIM \#602473). Deficiencies in several respiratory chain complexes have been reported in some of these pathologies, such as MOHBA (I and IV) [114], MGA (I and V) [115], D-2-OHGA (V and IV) [116], and EE (IV and II) [117]. Abnormal structure of mitochondria was also found in patients' samples with MHGA [118].

A growing body of experimental evidence indicates that impairment of redox homeostasis induced by the major organic acids accumulating in 3-HMGA contributes to the brain and liver pathophysiology [3]. Lipid and protein damage, along with a decrease of antioxidant defences, have been observed in patients' plasma and urine [3].

A recent transcriptome study in MCC-deficient patientderived fibroblasts revealed an altered gene expression profile indicative of mitochondrial dysfunction, decreased antioxidant response, and disruption of energy homeostasis [119]. This correlates with previous in vitro studies in the cerebral cortex of young rats in which the accumulated metabolites have been shown to disrupt energy homeostasis and cause lipid peroxidation [120].

\section{Homocystinuria}

In several IEMs, increased ROS causes pathophysiological oxidative damage that is not originated in the mitochondria. This is the case of homocystinuria in which excess homocysteine (Hcy) directly promotes ROS formation. Hcy is a sulphur-containing amino acid derived from the demethylation of the essential amino acid methionine. Autoxidation of two Hcy molecules yields the oxidized disulphide homocystine, two protons and two electrons, generating ROS in the form of $\mathrm{O}_{2}{ }^{-}$, hydrogen radical, or $\mathrm{H}_{2} \mathrm{O}_{2}$ [121]. Formation of mixed disulphides also contributes to the additional formation of ROS [121]. In addition to its inherent reactivity, specific mechanisms such as reduction in bioavailable nitric oxide and decreased glutathione peroxidase activity may play a role in $\mathrm{Hcy}$-induced oxidative stress [122, 123]. Plasma Hcy has been known for decades as a risk marker for cardiovascular disease, which is explained by Hcy-induced oxidative damage in endothelial cells [124]. Induction of NADPH oxidase or downregulation of thioredoxin expression have been postulated as underlying mechanisms [125]. Elevated homocysteine concentration has also been proposed as a risk factor for neurodegenerative diseases inducing neurological dysfunction via oxidative stress [126].

Hcy metabolism stands at the intersection of two pathways: remethylation to methionine and transsulfuration to cystathionine [127]. Inherited homocystinurias have in common accumulation of homocysteine with subsequent neurotoxicity. This group of diseases encompasses two distinctive clinical entities: classical homocystinuria due to 
cystathionine $\beta$-synthase (CBS) deficiency (transsulfuration pathway) and the rare inborn errors of cobalamin and folate metabolism (remethylation pathway) [128] (Figure 1).

3.1. Homocystinuria due to Cystathionine $\beta$-Synthase Deficiency. Deficiency of cystathionine $\beta$-synthase (CBS, MIM \#236200) is a disorder of methionine metabolism leading to elevated methionine and an abnormal accumulation of Hcy and its metabolites (homocystine, homocysteinecysteine complex, and others) in the blood and urine. In the case of excess cellular methionine levels, the transsulfuration pathway plays an important role in Hcy metabolism and converts Hcy into cystathionine with the help of the enzyme CBS (Figure 1). The cystathionine formed is then converted into cysteine by cystathionine $\gamma$-lyase. Although the pathophysiology of CBS deficiency is not yet well established, Hcy excess or cysteine deficiency rather than the accumulation of methionine is more likely to cause the pathogenesis of this disease [127]. CBS deficiency is clinically characterized by heterogeneous clinical manifestations in various organs and tissues, such as thinning and lengthening of the long bones, osteoporosis, dislocation of the ocular lens, thromboembolism, and mental retardation [127].

Oxidative stress may play an important role in the pathophysiology of homocystinuria, as deduced from studies carried out in patients and animal models [129]. Protein, lipid, and DNA oxidative damage and decreased antioxidant defences have been found in patients [130-132]. Increases in lipid, protein, and DNA oxidative damage biomarkers have been reported in CBS-deficient mice [133] and in samples from animal models subjected to Hcy administration $[134,135]$.

3.2. Homocystinuria due to Remethylation Defects. Remethylation disorders are due to deficiencies of enzymes involved in the remethylation of Hcy to methionine causing homocystinuria. They include defects in methionine synthase (MTR, MIM \#156570), methionine synthase reductase (MTRR, MIM \#602568), MMADHC 5(MIM \#611935), and 5,10-methylene tetrahydrofolate reductase enzyme (MTHFR, MIM \#236250) (Figure 1) [136]. Biochemically, these disorders are characterized by Hcy elevation and low-to-normal methionine levels, without urinary methylmalonic acid excretion. Patients present severe clinical symptoms, which are mainly neurological for MTHFR deficiency and neurohematological for MTR and MTRR defects [136]. Two major mechanisms have been proposed to explain the pathogenesis of these diseases: (i) defective methionine synthesis (with a consequent drop in Sadenosylmethionine production and deficiencies of numerous methylation reactions leading to hypomyelination in the central nervous system) [137] and (ii) Hcy excess [127].

Patient-derived fibroblasts with defects in MTR, MTRR, or MTHFR show a significant increase in ROS content, in MnSOD levels, in the rate of apoptosis, and in the levels of the active phosphorylated forms of p38 and JNK stress kinases [7]. Increased mRNA and protein levels of Herp, Grp78, IP ${ }_{3} \mathrm{R} 1$, pPERK, ATF4, CHOP, asparagine synthase,
GADD45, and MAM-associated proteins were also found in these cells indicating an increase in endoplasmic reticulum stress and subsequent mitochondrial calcium overload. In addition, an activation of autophagy process and a substantial degradation of altered mitochondria by mitophagy were detected in patient-derived fibroblasts [138].

\section{Challenges in ROS Detection}

ROS production plays an important role in physiological and pathological processes. In order to understand how they contribute to these cellular processes, it is essential to identify the specific reactive species produced, levels, and subcellular localization. ROS lifetime ranges from nanoseconds to seconds due to their high reactivity and depends on numerous clearance mechanisms such as the intracellular antioxidant levels [139]. Steady state concentration of mitochondrial $\mathrm{O}_{2}^{-}$and $\mathrm{H}_{2} \mathrm{O}_{2}$ have been estimated to be in the picomolar and low nanomolar ranges, respectively. ROS detection thereby requires specific molecular probes that promptly interact with ROS to compete with antioxidants and generate stable products, which can be accurately quantified. However, their very short life span and extremely low concentration and instability, as well as the large diversity of possible chemical reactions, make ROS assessment challenging. Excellent reviews by different authors cover the limitations, progress, and perspectives of the most widely used tools and new approaches described for monitoring ROS detection [139-142]. In this section, we focus on some of the most common detection strategies used to asses oxidative stress in vitro and in vivo in organic acidurias, pointing out some of their methodological constrains and concerns.

Assessment of ROS levels has largely relied on the detection of end products [143]. The small cell-permeable probes $2^{\prime}, 7^{\prime}$-dichlorodihydrofluorescein $\left(\mathrm{H}_{2} \mathrm{DCF}\right)$ and dihydroethidium (DHE) are the most commonly used molecules for intracellular ROS detection of $\mathrm{H}_{2} \mathrm{O}_{2}$ and $\mathrm{O}_{2}{ }^{-}$, respectively. Despite the fact that $\mathrm{H}_{2} \mathrm{DCF}$ is one of the most commonly used probes, many concerns have been raised regarding its mechanism of action and specificity [144]. For instance, it is known that a host of ROS species such as nitric oxide, peroxynitrite anions (ONOO-), and even organic hydroperoxides can oxidize $\mathrm{H}_{2}$ DCF. In addition, redox reactions with DCF and DCFH may lead to generation of the DCF-free radical anion [145], which produces $\mathrm{O}_{2}{ }^{-}$and reacts with antioxidants such as thiols and ascorbate [146]. Intracellular ROS generation throughout oxidation of $\mathrm{H}_{2} \mathrm{DCF}$ has been detected in PA, cobalamin disorders (cblB, cblC, cblE, and cblG), MTHFR deficiency, and MSUD patient-derived fibroblasts [26, 42, 98]. Recently, mitochondrial ROS generation has been measured in the cortex of a mouse model of MMA (induced by MMA and ammonia) [147]. DCF measurements should be carefully considered, and extra controls for the assessment of ROS production are required. Thus, DCF has been suggested as a general oxidative stress indicator instead of serving as a specific proof of $\mathrm{H}_{2} \mathrm{O}_{2}$ production.

DHE has been extensively used for intracellular $\mathrm{O}_{2}{ }^{-}$measurements together with its mitochondrial-targeted analog (MitoSOX). The specificity of DHE and its oxidative 
products has been a matter of debate for decades [144]. DHE can form two fluorescent products. One is ethidium, which is formed by nonspecific redox reactions, while the other is 2hydroxyethidium (2-OH-E+), a specific adduct of $\mathrm{O}_{2}{ }^{-}$ [148]. The fluorescent spectra of ethidium and 2-OH-E+ overlap, and it is therefore difficult to accurately measure only $2-\mathrm{OH}-\mathrm{E}+$ by simple fluorescent detection methods. However, careful use of specific wavelengths of excitation allows separation of these to signals [149]. DHE staining has been used to measure $\mathrm{O}_{2}{ }^{-}$production in brain and heart cryosections of $\mathrm{Pcca}^{-/-}$(A138T) mice [5]. MitoSOX has been used to measure the basal production of mitochondrial $\mathrm{O}_{2}{ }^{-}$in cobalamin disorders (cblB and cblE) and in BCKDK-deficient patientderived fibroblasts $[6,7,54]$. However, the combination of DHE and analytical methods such as high-pressure liquid chromatography and mass spectrometry is essential to validate $\mathrm{DHE}$ as a technique to quantify $\mathrm{O}_{2}{ }^{-}[150]$.

In order to have a better understanding of ROS mitochondrial regulation and its role in oxidative damage and redox signaling, new approaches to measure mitochondrial ROS levels within living organisms are essential. Logan et al. developed a radiometric mass spectrometry probe approach. This method is based on the use of the MitoB probe, which comprises a triphenylphosphonium (TPP) cation driving its accumulation inside the mitochondria, conjugated to an arylboronic acid that reacts with $\mathrm{H}_{2} \mathrm{O}_{2}$ to form a phenol MitoP. Once MitoB is administrated to the animal, the probe is modified in vivo by ROS to generate an exomarker product (MitoP). The MitoB/MitoP ratio allows assessment of the levels of ROS in vivo $[151,152]$. This technique has been applied to the mouse model of PA, to measure relative levels of $\mathrm{H}_{2} \mathrm{O}_{2}$ in the liver and heart [5]. The main advantages of this approach are its high selectivity, in vivo localization, and the use of analytical techniques such as LC-MS/MS to identify and quantify exomarkers. However, some significant weaknesses are also observed such as its inherently invasive methodology and the time-consuming nature of sample preparation.

Despite the progress achieved in the field of ROS detection, there is a clear need to develop noninvasive, in vivo, and more selective methods to address many important questions related to the role of altered redox homeostasis in the pathology of IEMs.

\section{Redox-Based Therapeutic Approaches}

Most IEMs in which altered redox homeostasis and oxidative damage have been documented as described in this work do not have an effective treatment. Therapy mainly consists on protein restriction and supplementation with special formulas that provide the necessary nutrients for adequate growth and development, as well as the administration of specific compounds aiming to detoxify the accumulated metabolites or to enhance residual enzymatic activities [10, 153]. However, the clinical outcome overall remains unsatisfactory, as multiorgan complications (neurological, cardiac, renal, hematological, and gastrointestinal, among others) persist. In this scenario, antioxidants and mitochondrial activators/ protectors may be beneficial to prevent or ameliorate oxidative damage contributing to organ pathophysiology.

Chemically, antioxidants are reduced compounds that directly react with ROS avoiding the oxidation of a third molecule. The in vitro mode of action of nutraceutical and synthetic antioxidants is mainly based on their capacity to act as free radical scavengers. However, in vivo antioxidant response depends on distribution, absorption, metabolism, and excretion of the compound, hampering a direct action on ROS. In fact, it is now clear that in vivo antioxidants provide indirect cellular and tissue protection against oxidative damage by targeting NRF2 and NAD-dependent protein deacetylase sirtuin-1 (SIRT1) [154, 155], modulating the antioxidant response (Figure 2).

NRF2 transcription factor regulates cellular homeostasis by binding to regulatory DNA regions known as electrophile response elements or EpRE (previously named antioxidant regulatory elements (AREs)). Under oxidative stress, NRF2 translocates to the nucleus, binds to EpRE sequences, and promotes the transcription of antioxidant enzymes, genes of GSH metabolism, and NADPH regeneration (Figure 2) [156].

Antioxidant response can also be driven by SIRT1, a class I histone deacetylase that mediates deacetylation in a NAD+-dependent manner. SIRT1 is mainly localized in the nucleus where, apart from histones, it can interact with and deacetylate peroxisome proliferator-activated receptor gamma coactivator 1-alpha $(\mathrm{PGC1} \alpha)$ and Forkhead box protein O3 (FOXOa3) $[157,158]$. Deacetylated PGC1 $\alpha$ and FOXOa3 have an increased transcriptional activity and promote mitochondrial biogenesis and expression of antioxidant genes (Figure 2). Therefore, NRF2 and SIRT1 activation by polyphenolic compounds represent the main targets for antioxidant therapy to reduce oxidative damage.

Several studies have evaluated different antioxidant compounds as adjuvant therapy in a number of IEMs. The brain is highly sensitive to free radicals due to its high energetic demands, its high lipid content, and its low regeneration capacity. In this context, oxidative damage has been proposed as one of the mechanisms involved in the development of the characteristic neurological symptoms of PA, MMA, MSUD, GAI, and homocystinuria patients. Even though the transport rate of antioxidants through the blood brain barrier is low, different studies have reported a reversion of brain damage after antioxidant treatments in these diseases [159-162].

In organic acidurias, a beneficial effect of L-carnitine supplementation with in vitro antioxidant properties has been described [13, 163]. L-carnitine shows antioxidant capacities as it can act as ROS scavenger and reduce lipid peroxidation to an extent comparable to that seen with $\alpha$-tocopherol [164]. It also protects endogenous antioxidant enzymes from peroxidative damage and induces elevated cellular GSH [31]. L-carnitine supplementation is routinely included in the treatment of PA and MMA, to prevent a secondary carnitine deficiency and to promote detoxification of propionic and methylmalonic acids, which are excreted in the form of carnitine esters. Evaluation of oxidative stress parameters in urine from PA and MMA patients during treatment with L-carnitine- and protein-restricted diet showed 


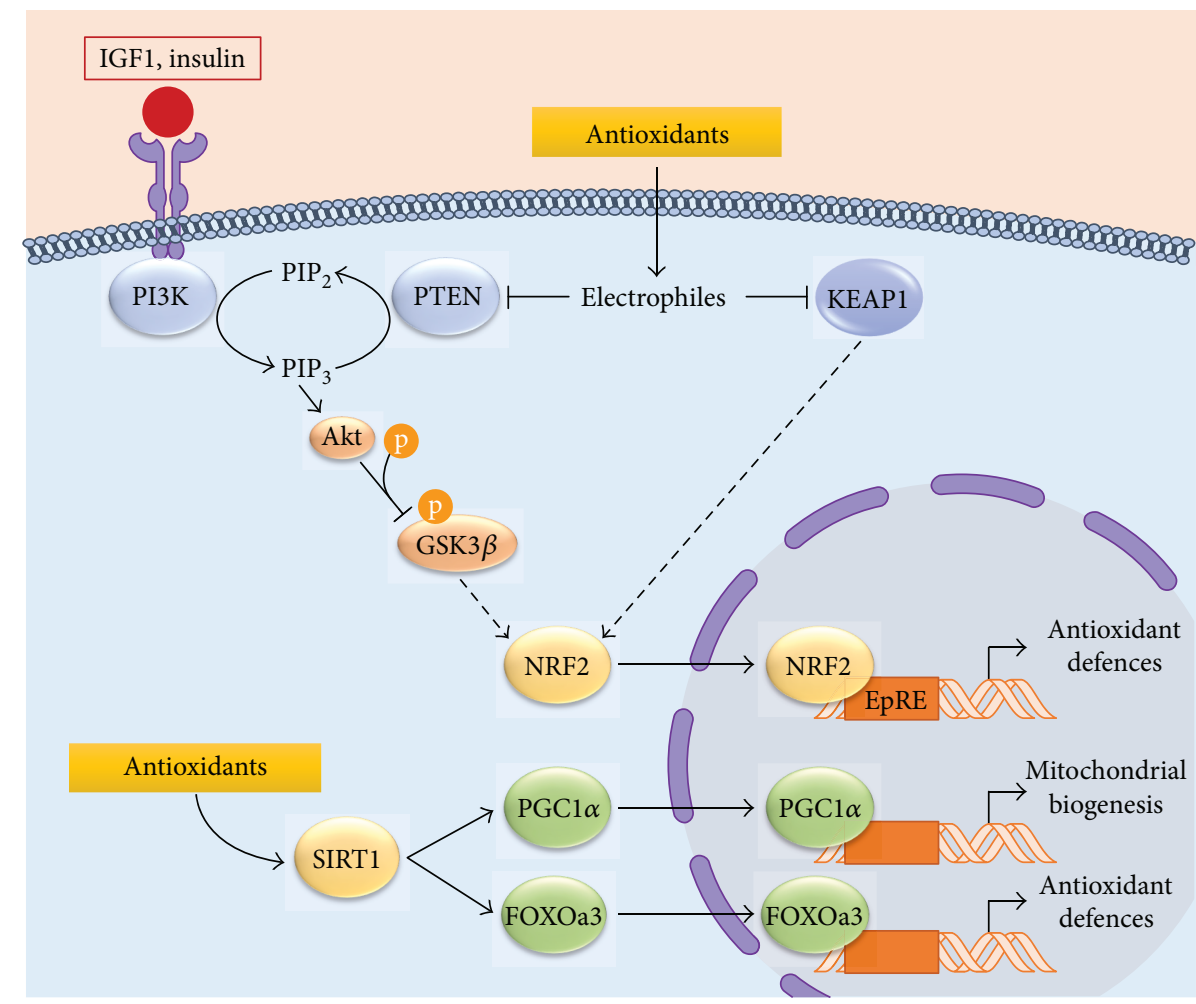

FIGURE 2: NRF2- and SIRT1-signaling pathways targeted by antioxidant compounds. Under normal homeostatic conditions, NRF2 transcriptional activity is inhibited by KEAP1 and PTEN. KEAP1 directly interacts with NRF2 and mediates its ubiquitination and subsequent proteasomal degradation. Furthermore, PTEN eliminates 3-phosphoinositides $\left(\mathrm{PIP}_{3}\right)$ required for AKT activation, thus leading to GSK3 $\beta$ activation and NRF2 phosphorylation. In vivo antioxidants act as electrophiles that modify and inhibit KEAP1 and PTEN. When KEAP1 is oxidized, its interaction with NRF2 is disrupted and NRF2 half-life increases. Electrophiles also inhibit the redoxsensitive phosphatase PTEN, allowing $\mathrm{PIP}_{3}$ accumulation, AKT activation, and GSK3 $\beta$ inactivation. In these conditions, NRF2 is translocated to the nucleus where it binds to the electrophile response element (EpRE) and drives the expression of antioxidant defence genes. Additionally, antioxidant compounds modulate SIRT1 pathway favouring the antioxidant defence response and mitochondrial biogenesis by activation of FOXOa3 and PGC1 $\alpha$ transcription factors, respectively.

a significant reduction of urinary and plasma biomarkers of oxidative damage to lipids and proteins $[13,163]$. Lcarnitine treatment was also shown to reduce plasma malondialdehyde concentrations and urine dityrosine and isoprostanes in patients with MSUD [51, 165].

In a MMA patient with optic neuropathy, $\mathrm{CoQ}_{10}$ treatment in conjunction with vitamin $\mathrm{E}$ resulted in improved visual acuity [166]. Interestingly, treatment of MMA mice with $\mathrm{CoQ}_{10}$ and vitamin $\mathrm{E}$ showed a significant amelioration in the loss of glomerular filtration rate and a normalization of plasma lipocalin-2 levels [89], indicating that the therapeutic effects are not restricted to the nervous system.

Recently, in vitro and in vivo studies in PA have also shown the positive effect of antioxidant treatment. Using patients' fibroblasts, different antioxidants (tiron, trolox, resveratrol, and MitoQ) significantly reduced $\mathrm{H}_{2} \mathrm{O}_{2}$ levels and regulated the expression of antioxidant enzymes [30]. In the hypomorphic mouse model of PA [167], oral treatment with resveratrol and MitoQ protected against lipid and DNA oxidative damage and induced the expression of antioxidant enzymes [32]. In addition to its antioxidant properties, resveratrol exerts different effects on mitochondrial function and dynamics [168], which clearly contributes to its beneficial effect in IEMs with mitochondrial ROS production, such as organic acidemias and respiratory chain defects [169].

A clinical trial (NCT01793090) is ongoing in patients with MMA cblC-type defect to investigate the safety and efficacy of the EPI-743 compound in relation to visual and neurological impairment. This molecule targets NADPH quinone oxidoreductase 1 , thus providing beneficial effects in diseases characterized by oxidative stress and alterations in glutathione redox balance, as is the case of mitochondrial diseases [170].

Taurine, a compound potentially reducing oxidative damage, is being tested in a clinical trial with homocystinuria (CBS-deficient) patients (NCT01192828). Taurine treatment in a mouse model of CBS deficiency reversed GSH depletion with potential beneficial effects [171].

Although the use of antioxidants as potential therapies holds great interest for many diseases (including IEMs) in which oxidative stress plays a pathophysiological role, there is to date limited clinical evidence of their efficacy, which may be related to pharmacokinetic and/or pharmacodynamics 


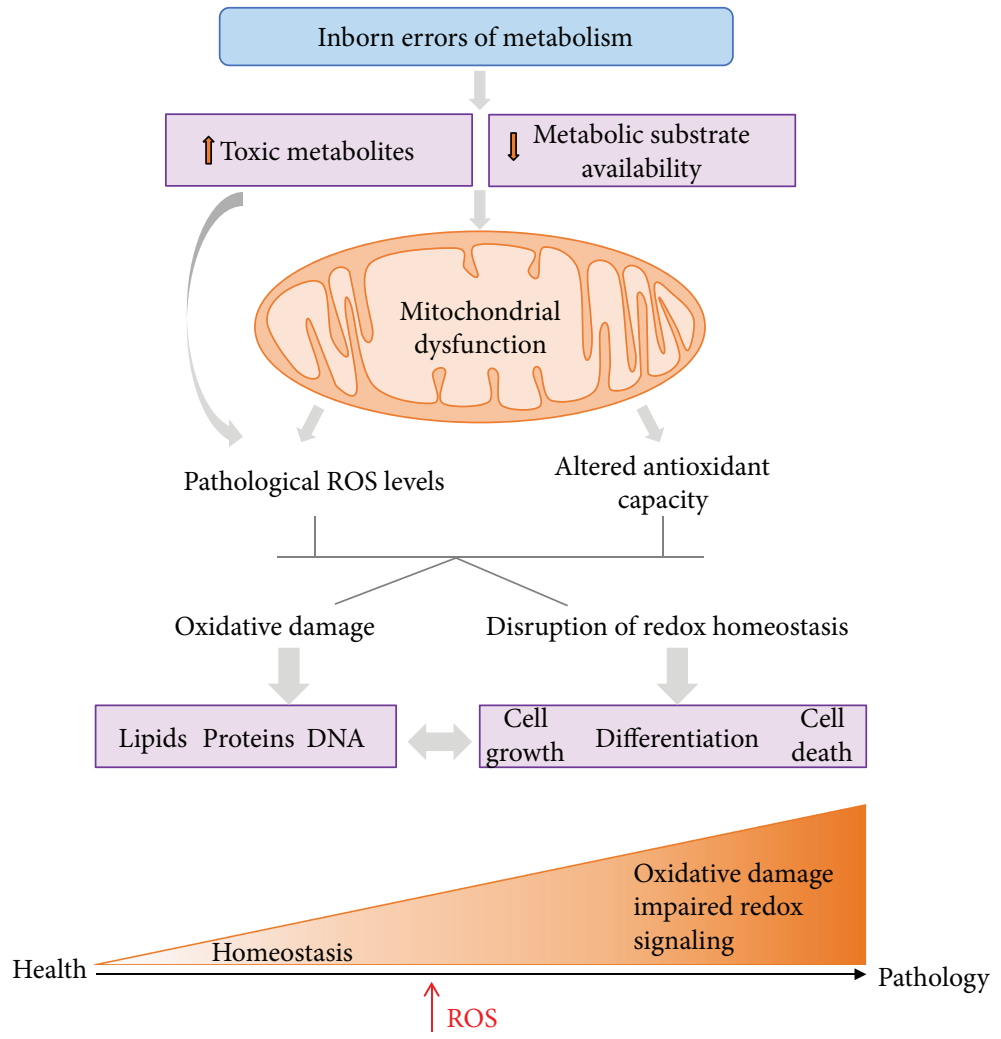

FIgURE 3: ROS and oxidative damage contribute to IEM physiopathology. The scheme shows the consequences of toxic metabolite accumulation in IEMs on mitochondrial function and ROS increase, which in turn results in oxidative damage and alteration in ROS signaling contributing to disease pathology.

reasons [172]. The intracellular levels of the specific antioxidant, its distribution throughout the body, together with the subcellular location are factors that influence the in vivo effects. Moreover, antioxidants react with free radicals with the same rate constant as other natural molecules, and their intracellular concentrations cannot overcome this kinetic limitation. Therefore, antioxidant effectiveness in vivo could be limited [154].

Throughout the years, different studies have shown both beneficial and adverse effects of antioxidant compounds. In vitro $\beta$-carotene shows antioxidant and antiinflammatory effects when administered at low doses whereas prooxidant and proinflammatory responses have been reported at higher concentrations [173, 174]. Similar contradictory results have been obtained in in vivo studies. While in a randomized trial dietary consumption of $\beta$-carotene and vitamins $\mathrm{C}$ and $\mathrm{E}$ did not affect cancer incidence and mortality [175], higher doses of these compounds did not show any health beneficial effect. In fact, they could significantly increase the risk of mortality (reviewed in $[176,177])$. ROS are required at physiological concentrations for optimal cellular homeostasis [178]. Therefore, free radical scavenging by antioxidants could be undesirable as disruption of redox balance would lead to cellular dysfunction and originate different side effects.

New indirect antioxidant approaches targeting redox enzymes or NRF2 agonists that upregulate cellular antioxidant defences are promising alternatives that warrant further investigation [155].

\section{Conclusions and Future Perspectives}

The pathophysiological role of ROS in IEMs has been firmly established in many studies revealing oxidative damage to proteins, lipids, and DNA in patients' samples and animal models, correlating with clinical and biochemical parameters (Figure 3). Consequently, different antioxidants have been successfully used in cellular and animal models of IEMs [30-32, 89, 98]. Despite these positive results, for other diseases, there is no clear conclusion on the efficacy of antioxidant treatments in human clinical trials, which limits the expectations also for IEMs.

In the past years, we have gained deeper insights into the complex and dynamic roles of ROS signals in normal and pathological processes, yet the field of redox signaling in IEMs is still in its infancy. Novel technologies such as redox proteomics [179] or transgenic animals expressing redox reporters [180] are revolutionising the field and represent excellent tools to investigate redox regulatory networks in disease. They may also provide insight to explain the poor performance of antioxidant therapies in clinical trials. In many IEMs, mitochondrial ROS-targeting agents that accumulate in the mitochondria and compounds activating or improving mitochondrial function may constitute a 
valid approach. Further preclinical research is warranted to investigate basic mechanisms of redox signaling and their implications for IEM disease progression, as well as exploring the benefits of targeted antioxidant therapies.

\section{Disclosure}

Lorena Gallego-Villar's present address is the Department of Pediatrics and Adolescent Medicine, Division of Pediatric Hematology and Oncology, University Medical Centre Freiburg, Freiburg, Germany. Alfonso Oyarzábal's present address is the Institut de Recerca Pediàtrica-Hospital Sant Joan de Déu (IRP-HSJD), CIBERER, Barcelona, Spain

\section{Conflicts of Interest}

The authors declare that they have no conflicts of interest.

\section{Acknowledgments}

This work was supported by the Spanish Ministry of Economy and Competitiveness and the European Regional Development Fund (Grant nos. SAF2016-76004-R and PI16/00573) and by Fundación Isabel Gemio. Centro de Biología Molecular Severo Ochoa receives an institutional grant from Fundación Ramón Areces.

\section{References}

[1] R. K. J. Olsen, N. Cornelius, and N. Gregersen, "Redox signalling and mitochondrial stress responses; lessons from inborn errors of metabolism," Journal of Inherited Metabolic Disease, vol. 38, no. 4, pp. 703-719, 2015.

[2] M. Wajner, A. Latini, A. T. S. Wyse, and C. S. Dutra-Filho, "The role of oxidative damage in the neuropathology of organic acidurias: insights from animal studies," Journal of Inherited Metabolic Disease, vol. 27, no. 4, pp. 427-448, 2004.

[3] G. Leipnitz, C. R. Vargas, and M. Wajner, "Disturbance of redox homeostasis as a contributing underlying pathomechanism of brain and liver alterations in 3-hydroxy-3-methylglutaryl-CoA lyase deficiency," Journal of Inherited Metabolic Disease, vol. 38, no. 6, pp. 1021-1028, 2015.

[4] M. Wajner and S. I. Goodman, "Disruption of mitochondrial homeostasis in organic acidurias: insights from human and animal studies," Journal of Inherited Metabolic Disease, vol. 43, no. 1, pp. 31-38, 2011.

[5] L. Gallego-Villar, A. Rivera-Barahona, C. Cuevas-Martin et al., "In vivo evidence of mitochondrial dysfunction and altered redox homeostasis in a genetic mouse model of propionic acidemia: implications for the pathophysiology of this disorder," Free Radical Biology and Medicine, vol. 96, pp. 1-12, 2016.

[6] E. Richard, A. Alvarez-Barrientos, B. Perez, L. R. Desviat, and M. Ugarte, "Methylmalonic acidaemia leads to increased production of reactive oxygen species and induction of apoptosis through the mitochondrial/caspase pathway," The Journal of Pathology, vol. 213, no. 4, pp. 453-461, 2007.

[7] E. Richard, L. R. Desviat, M. Ugarte, and B. Perez, "Oxidative stress and apoptosis in homocystinuria patients with genetic remethylation defects," Journal of Cellular Biochemistry, vol. 114, no. 1, pp. 183-191, 2013.
[8] E. Morava, S. Rahman, V. Peters, M. R. Baumgartner, M. Patterson, and J. Zschocke, "Quo vadis: the re-definition of "inborn metabolic diseases"," Journal of Inherited Metabolic Disease, vol. 38, no. 6, pp. 1003-6, 2015.

[9] R. K. J. Olsen, N. Cornelius, and N. Gregersen, "Genetic and cellular modifiers of oxidative stress: what can we learn from fatty acid oxidation defects?," Molecular Genetics and Metabolism, vol. 110, pp. S31-S39, 2013.

[10] E. Richard, P. Rodriguez-Pombo, L. R. Desviat et al., "Mitochondrial organic acidurias. Part II: mitochondrial dysfunction," in Mitochondrial Pathophysiology, F. Palau and S. Cadenas, Eds., pp. 173-191, Research Signpost, Kerala, India, 2011.

[11] E. L. Streck, G. A. Czapski, and C. Goncalves da Silva, "Neurodegeneration, mitochondrial dysfunction, and oxidative stress," Oxidative Medicine and Cellular Longevity, vol. 2013, Article ID 826046, 2 pages, 2013.

[12] P. J. Mc Guire, A. Parikh, and G. A. Diaz, "Profiling of oxidative stress in patients with inborn errors of metabolism," Molecular Genetics and Metabolism, vol. 98, no. 1-2, pp. 173-180, 2009.

[13] G. S. Ribas, G. B. Biancini, C. Mescka et al., "Oxidative stress parameters in urine from patients with disorders of propionate metabolism: a beneficial effect of L-carnitine supplementation," Cellular and Molecular Neurobiology, vol. 32, no. 1, pp. 77-82, 2012.

[14] R. J. Mailloux, S. L. McBride, and M. E. Harper, "Unearthing the secrets of mitochondrial ROS and glutathione in bioenergetics," Trends in Biochemical Sciences, vol. 38, no. 12, pp. 592-602, 2013.

[15] A. Bindoli and M. P. Rigobello, "Principles in redox signaling: from chemistry to functional significance," Antioxidants \& Redox Signaling, vol. 18, no. 13, pp. 1557-1593, 2013.

[16] T. Finkel, "Signal transduction by mitochondrial oxidants," Journal of Biological Chemistry, vol. 287, no. 7, pp. 44344440, 2012.

[17] N. Ballatori, S. M. Krance, S. Notenboom, S. Shi, K. Tieu, and C. L. Hammond, "Glutathione dysregulation and the etiology and progression of human diseases," Biological Chemistry, vol. 390, no. 3, pp. 191-214, 2009.

[18] H. Salmi, J. V. Leonard, and R. Lapatto, "Patients with organic acidaemias have an altered thiol status," Acta Paediatrica, vol. 101, no. 11, pp. e505-e508, 2012.

[19] A. Pastore, D. Martinelli, F. Piemonte et al., "Glutathione metabolism in cobalamin deficiency type C (cblC)," Journal of Inherited Metabolic Disease, vol. 37, no. 1, pp. 125-129, 2014.

[20] K. R. Atkuri, T. M. Cowan, T. Kwan et al., "Inherited disorders affecting mitochondrial function are associated with glutathione deficiency and hypocitrullinemia," Proceedings of the National Academy of Sciences, vol. 106, no. 10, pp. 3941-3945, 2009.

[21] M. P. Murphy, "Mitochondrial thiols in antioxidant protection and redox signaling: distinct roles for glutathionylation and other thiol modifications," Antioxidants \& Redox Signaling, vol. 16, no. 6, pp. 476-495, 2012.

[22] M. C. Sobotta, W. Liou, S. Stöcker et al., "Peroxiredoxin-2 and STAT3 form a redox relay for $\mathrm{H} 2 \mathrm{O} 2$ signaling," Nature Chemical Biology, vol. 11, no. 1, pp. 64-70, 2015.

[23] F. Yin, H. Sancheti, Z. Liu, and E. Cadenas, "Mitochondrial function in ageing: coordination with signalling and 
transcriptional pathways," The Journal of Physiology, vol. 594, no. 8, pp. 2025-2042, 2016.

[24] H. Wu, H. Wei, S. A. Sehgal, L. Liu, and Q. Chen, "Mitophagy receptors sense stress signals and couple mitochondrial dynamic machinery for mitochondrial quality control," Free Radical Biology and Medicine, vol. 100, pp. 199-209, 2016.

[25] A. Boneh, "Signal transduction in inherited metabolic disorders: a model for a possible pathogenetic mechanism," Journal of Inherited Metabolic Disease, vol. 38, no. 4, pp. 729-740, 2015.

[26] L. Gallego-Villar, C. Perez-Cerda, B. Perez et al., "Functional characterization of novel genotypes and cellular oxidative stress studies in propionic acidemia," Journal of Inherited Metabolic Disease, vol. 36, no. 5, pp. 731-740, 2013.

[27] P. Alcaide, B. Merinero, P. Ruiz-Sala et al., "Defining the pathogenicity of creatine deficiency syndrome," Human Mutation, vol. 32, no. 3, pp. 282-291, 2011.

[28] H. Rocha, R. Ferreira, J. Carvalho et al., "Characterization of mitochondrial proteome in a severe case of ETF-QO deficiency," Journal of Proteomics, vol. 75, no. 1, pp. 221-228, 2011.

[29] J. Lopez-Erauskin, S. Fourcade, J. Galino et al., "Antioxidants halt axonal degeneration in a mouse model of X-adrenoleukodystrophy," Annals of Neurology, vol. 70, no. 1, pp. 8492, 2011.

[30] L. Gallego-Villar, B. Perez, M. Ugarte, L. R. Desviat, and E. Richard, "Antioxidants successfully reduce ROS production in propionic acidemia fibroblasts," Biochemical and Biophysical Research Communications, vol. 452, no. 3, pp. 457-461, 2014.

[31] G. S. Ribas, C. R. Vargas, and M. Wajner, "L-carnitine supplementation as a potential antioxidant therapy for inherited neurometabolic disorders," Gene, vol. 533, no. 2, pp. 469476, 2014.

[32] A. Rivera-Barahona, E. Alonso-Barroso, B. Perez, M. P. Murphy, E. Richard, and L. R. Desviat, "Treatment with antioxidants ameliorates oxidative damage in a mouse model of propionic acidemia," Molecular Genetics and Metabolism, vol. 122, no. 1-2, pp. 43-50, 2017.

[33] G. Hayashi and G. Cortopassi, "Oxidative stress in inherited mitochondrial diseases," Free Radical Biology and Medicine, vol. 88, no. Part A, pp. 10-17, 2015.

[34] J. E. Sperringer, A. Addington, and S. M. Hutson, "Branchedchain amino acids and brain metabolism," Neurochemical Research, vol. 42, no. 6, pp. 1697-1709, 2017.

[35] J. M. Han, S. J. Jeong, M. C. Park et al., "Leucyl-tRNA synthetase is an intracellular leucine sensor for the mTORC1signaling pathway," Cell, vol. 149, no. 2, pp. 410-424, 2012.

[36] K. A. Anderson, F. K. Huynh, K. Fisher-Wellman et al., "SIRT4 is a lysine deacylase that controls leucine metabolism and insulin secretion," Cell Metabolism, vol. 25, no. 4, pp. 838-855.e15, 2017.

[37] D. T. Chuang and V. E. Shi, "Maple syrup urine diesease (branched chain ketoaciduria)," in The Metabolic and Molecular Basis of Inherited Disease, C. R. Scriver, A. L. Beaudet, W. Sly, and D. Valle, Eds., pp. 1971-2005, McGraw-Hill, New York, 2001.

[38] G. Novarino, P. El-Fishawy, H. Kayserili et al., "Mutations in BCKD-kinase lead to a potentially treatable form of autism with epilepsy," Science, vol. 338, no. 6105, pp. 394-397, 2012.
[39] A. Garcia-Cazorla, A. Oyarzabal, J. Fort et al., "Two novel mutations in the BCKDK (branched-chain keto-acid dehydrogenase kinase) gene are responsible for a neurobehavioral deficit in two pediatric unrelated patients," Human Mutation, vol. 35, no. 4, pp. 470-477, 2014.

[40] G. R. D. Villani, G. Gallo, E. Scolamiero, F. Salvatore, and M. Ruoppolo, "“Classical organic acidurias": diagnosis and pathogenesis," Clinical and Experimental Medicine, vol. 17, no. 3, pp. 305-323, 2017.

[41] R. A. Harris, M. Joshi, and N. H. Jeoung, "Mechanisms responsible for regulation of branched-chain amino acid catabolism," Biochemical and Biophysical Research Communications, vol. 313, no. 2, pp. 391-396, 2004.

[42] A. Oyarzabal, M. Martinez-Pardo, B. Merinero et al., "A novel regulatory defect in the branched-chain $\alpha$-keto acid dehydrogenase complex due to a mutation in the PPM1K gene causes a mild variant phenotype of maple syrup urine disease," Human Mutation, vol. 34, no. 2, pp. 355-362, 2013.

[43] A. Sitta, G. S. Ribas, C. P. Mescka, A. G. Barschak, M. Wajner, and C. R. Vargas, "Neurological damage in MSUD: the role of oxidative stress," Cellular and Molecular Neurobiology, vol. 34, no. 2, pp. 157-165, 2014.

[44] P. de Lima Pelaez, C. Funchal, S. O. Loureiro et al., "Branched-chain amino acids accumulating in maple syrup urine disease induce morphological alterations in C6 glioma cells probably through reactive species," International Journal of Developmental Neuroscience, vol. 25, no. 3, pp. 181-189, 2007.

[45] W. J. Zinnanti, J. Lazovic, K. Griffin et al., "Dual mechanism of brain injury and novel treatment strategy in maple syrup urine disease," Brain, vol. 132, Part 4, pp. 903-918, 2009.

[46] T. Friedrich, A. M. Lambert, M. A. Masino, and G. B. Downes, "Mutation of zebrafish dihydrolipoamide branched-chain transacylase E2 results in motor dysfunction and models maple syrup urine disease," Disease Models \& Mechanisms, vol. 5, no. 2, pp. 248-258, 2012.

[47] R. Bridi, J. Araldi, M. B. Sgarbi et al., "Induction of oxidative stress in rat brain by the metabolites accumulating in maple syrup urine disease," International Journal of Developmental Neuroscience, vol. 21, no. 6, pp. 327-332, 2003.

[48] R. Bridi, C. A. Braun, G. K. Zorzi et al., " $\alpha$-Keto acids accumulating in maple syrup urine disease stimulate lipid peroxidation and reduce antioxidant defences in cerebral cortex from young rats," Metabolic Brain Disease, vol. 20, no. 2, pp. 155-167, 2005.

[49] O. Zhenyukh, E. Civantos, M. Ruiz-Ortega et al., "High concentration of branched-chain amino acids promotes oxidative stress, inflammation and migration of human peripheral blood mononuclear cells via mTORC1 activation," Free Radical Biology and Medicine, vol. 104, pp. 165-177, 2017.

[50] A. G. Barschak, A. Sitta, M. Deon et al., "Oxidative stress in plasma from maple syrup urine disease patients during treatment," Metabolic Brain Disease, vol. 23, no. 1, pp. 7180, 2008.

[51] C. P. Mescka, C. A. Y. Wayhs, C. S. Vanzin et al., "Protein and lipid damage in maple syrup urine disease patients: L-carnitine effect," International Journal of Developmental Neuroscience, vol. 31, no. 1, pp. 21-24, 2013.

[52] C. P. Mescka, G. Guerreiro, B. Donida et al., "Investigation of inflammatory profile in MSUD patients: benefit of 
L-carnitine supplementation," Metabolic Brain Disease, vol. 30, no. 5, pp. 1167-1174, 2015.

[53] G. Lu, S. Ren, P. Korge et al., "A novel mitochondrial matrix serine/threonine protein phosphatase regulates the mitochondria permeability transition pore and is essential for cellular survival and development," Genes \& Development, vol. 21, no. 7, pp. 784-796, 2007.

[54] A. Oyarzabal, I. Bravo-Alonso, M. Sanchez-Arago et al., "Mitochondrial response to the BCKDK-deficiency: some clues to understand the positive dietary response in this form of autism," Biochimica et Biophysica Acta (BBA) - Molecular Basis of Disease, vol. 1862, no. 4, pp. 592-600, 2016.

[55] J. Kaplon, L. Zheng, K. Meissl et al., "A key role for mitochondrial gatekeeper pyruvate dehydrogenase in oncogeneinduced senescence," Nature, vol. 498, no. 7452, pp. 109$112,2013$.

[56] C. L. Quinlan, R. L. S. Goncalves, M. Hey-Mogensen, N. Yadava, V. I. Bunik, and M. D. Brand, "The 2-oxoacid dehydrogenase complexes in mitochondria can produce superoxide/hydrogen peroxide at much higher rates than complex I," Journal of Biological Chemistry, vol. 289, no. 12, pp. 8312-8325, 2014.

[57] W. A. Fenton, R. A. Gravel, and L. E. Rosenberg, "Disorders of propionate and methylmalonate metabolism," in The Metabolic and Molecular Bases of Inherited Disease, C. R. Scriver, A. L. Beaudet, W. Sly, and D. Valle, Eds., pp. 2165-2190, McGraw-Hill, New York, 2001.

[58] L. Pena and B. K. Burton, "Survey of health status and complications among propionic acidemia patients," American Journal of Medical Genetics Part A, vol. 158A, no. 7, pp. 1641-1646, 2012.

[59] L. Pena, J. Franks, K. A. Chapman et al., "Natural history of propionic acidemia," Molecular Genetics and Metabolism, vol. 105, no. 1, pp. 5-9, 2012.

[60] S. Kölker, A. G. Cazorla, V. Valayannopoulos et al., “The phenotypic spectrum of organic acidurias and urea cycle disorders. Part 1: the initial presentation," Journal of Inherited Metabolic Disease, vol. 38, no. 6, pp. 1041-1057, 2015.

[61] S. Kölker, V. Valayannopoulos, A. B. Burlina et al., "The phenotypic spectrum of organic acidurias and urea cycle disorders. Part 2: the evolving clinical phenotype," Journal of Inherited Metabolic Disease, vol. 38, no. 6, pp. 10591074, 2015.

[62] S. C. Grünert, S. Müllerleile, L. De Silva et al., "Propionic acidemia: clinical course and outcome in 55 pediatric and adolescent patients," Orphanet Journal of Rare Diseases, vol. 8, no. 1, p. 6, 2013.

[63] J. Baruteau, I. Hargreaves, S. Krywawych et al., "Successful reversal of propionic acidaemia associated cardiomyopathy: evidence for low myocardial coenzyme Q10 status and secondary mitochondrial dysfunction as an underlying pathophysiological mechanism," Mitochondrion, vol. 17, pp. 150-156, 2014.

[64] M. Nizon, C. Ottolenghi, V. Valayannopoulos et al., "Longterm neurological outcome of a cohort of 80 patients with classical organic acidurias," Orphanet Journal of Rare Diseases, vol. 8, no. 1, p. 148, 2013.

[65] S. W. Sauer, J. G. Okun, G. F. Hoffmann, S. Koelker, and M. A. Morath, "Impact of short- and medium-chain organic acids, acylcarnitines, and acyl-CoAs on mitochondrial energy metabolism," Biochimica et Biophysica Acta (BBA) - Bioenergetics, vol. 1777, no. 10, pp. 1276-1282, 2008.
[66] N. Gregersen, "The specific inhibition of the pyruvate dehydrogenase complex from pig kidney by propionyl-CoA and isovaleryl-Co-A," Biochemical Medicine, vol. 26, no. 1, pp. 20-27, 1981.

[67] S. Cheema-Dhadli, C. C. Leznoff, and M. L. Halperin, "Effect of 2-methylcitrate on citrate metabolism: implications for the management of patients with propionic acidemia and methylmalonic aciduria," Pediatric Research, vol. 9, no. 12, pp. 905-908, 1975.

[68] D. A. Stumpf, J. Mcafee, J. K. Parks, and L. Eguren, "Propionate inhibition of succinate:CoA ligase (GDP) and the citric acid cycle in mitochondria," Pediatric Research, vol. 14, no. 10, pp. 1127-1131, 1980.

[69] H. Brunengraber and C. R. Roe, "Anaplerotic molecules: current and future," Journal of Inherited Metabolic Disease, vol. 29, no. 2-3, pp. 327-331, 2006.

[70] S. Nakao, Y. Moriya, S. Furuyama, R. Niederman, and H. Sugiya, "Propionic acid stimulates superoxide generation in human neutrophils," Cell Biology International, vol. 22, no. 5, pp. 331-337, 1998.

[71] F. K. Rigo, L. Pasquetti, C. R. M. Malfatti et al., "Propionic acid induces convulsions and protein carbonylation in rats," Neuroscience Letters, vol. 408, no. 2, pp. 151-154, 2006.

[72] F. U. Fontella, V. Pulrolnik, E. Gassen et al., "Propionic and L-methylmalonic acids induce oxidative stress in brain of young rats," Neuroreport, vol. 11, no. 3, pp. 541-544, 2000.

[73] Y. de Keyzer, V. Valayannopoulos, J. F. Benoist et al., "Multiple OXPHOS deficiency in the liver, kidney, heart, and skeletal muscle of patients with methylmalonic aciduria and propionic aciduria," Pediatric Research, vol. 66, no. 1, pp. 91-95, 2009.

[74] R. Mardach, M. A. Verity, and S. D. Cederbaum, "Clinical, pathological, and biochemical studies in a patient with propionic acidemia and fatal cardiomyopathy," Molecular Genetics and Metabolism, vol. 85, no. 4, pp. 286-290, 2005.

[75] M. A. Schwab, S. W. Sauer, J. G. Okun et al., "Secondary mitochondrial dysfunction in propionic aciduria: a pathogenic role for endogenous mitochondrial toxins," Biochemical Journal, vol. 398, no. 1, pp. 107-112, 2006.

[76] K. Fragaki, A. Cano, J. F. Benoist et al., "Fatal heart failure associated with CoQ10 and multiple OXPHOS deficiency in a child with propionic acidemia," Mitochondrion, vol. 11, no. 3, pp. 533-536, 2011.

[77] S. Yano, S. Yano, L. Li et al., "Infantile mitochondrial DNA depletion syndrome associated with methylmalonic aciduria and 3-methylcrotonyl-CoA and propionyl-CoA carboxylase deficiencies in two unrelated patients: a new phenotype of mtDNA depletion syndrome," Journal of Inherited Metabolic Disease, vol. 26, no. 5, pp. 481-488, 2003.

[78] D. R. Melo, A. J. Kowaltowski, M. Wajner, and R. F. Castilho, "Mitochondrial energy metabolism in neurodegeneration associated with methylmalonic acidemia," Journal of Bioenergetics and Biomembranes, vol. 43, no. 1, pp. 39-46, 2011.

[79] L. F. Pettenuzzo, C. Ferreira Gda, A. L. Schmidt, C. S. DutraFilho, A. T. Wyse, and M. Wajner, "Differential inhibitory effects of methylmalonic acid on respiratory chain complex activities in rat tissues," International Journal of Developmental Neuroscience, vol. 24, no. 1, pp. 45-52, 2006.

[80] S. R. Mirandola, D. R. Melo, P. F. Schuck, G. C. Ferreira, M. Wajner, and R. F. Castilho, "Methylmalonate inhibits succinate-supported oxygen consumption by interfering with 
mitochondrial succinate uptake," Journal of Inherited Metabolic Disease, vol. 31, no. 1, pp. 44-54, 2008.

[81] M. A. Morath, J. G. Okun, I. B. Muller et al., "Neurodegeneration and chronic renal failure in methylmalonic aciduria-a pathophysiological approach," Journal of Inherited Metabolic Disease, vol. 31, no. 1, pp. 35-43, 2008.

[82] S. Kolker and J. G. Okun, "Methylmalonic acid-an endogenous toxin?," Cellular and Molecular Life Sciences, vol. 62, no. 6, pp. 621-624, 2005.

[83] V. Valayannopoulos, L. Hubert, J. F. Benoist et al., "Multiple OXPHOS deficiency in the liver of a patient with CblA methylmalonic aciduria sensitive to vitamin B(12)," Journal of Inherited Metabolic Disease, vol. 32, no. 2, pp. 159-162, 2009.

[84] R. J. Chandler, P. M. Zerfas, S. Shanske et al., "Mitochondrial dysfunction in mut methylmalonic acidemia," The FASEB Journal, vol. 23, no. 4, pp. 1252-1261, 2009.

[85] K. Hayasaka, K. Metoki, T. Satoh et al., "Comparison of cytosolic and mitochondrial enzyme alterations in the livers of propionic or methylmalonic acidemia: a reduction of cytochrome oxidase activity," The Tohoku Journal of Experimental Medicine, vol. 137, no. 3, pp. 329-334, 1982.

[86] M. A. Cosson, G. Touati, F. Lacaille et al., "Liver hepatoblastoma and multiple OXPHOS deficiency in the follow-up of a patient with methylmalonic aciduria," Molecular Genetics and Metabolism, vol. 95, no. 1-2, pp. 107-109, 2008.

[87] M. Caterino, R. J. Chandler, J. L. Sloan et al., "The proteome of methylmalonic acidemia (MMA): the elucidation of altered pathways in patient livers," Molecular BioSystems, vol. 12, no. 2, pp. 566-574, 2016.

[88] E. Richard, L. Monteoliva, S. Juarez et al., "Quantitative analysis of mitochondrial protein expression in methylmalonic acidemia by two-dimensional difference gel electrophoresis," Journal of Proteome Research, vol. 5, no. 7, pp. 1602-1610, 2006.

[89] I. Manoli, J. R. Sysol, L. Li et al., "Targeting proximal tubule mitochondrial dysfunction attenuates the renal disease of methylmalonic acidemia," Proceedings of the National Academy of Sciences, vol. 110, no. 33, pp. 13552-13557, 2013.

[90] Z. K. Zsengeller, N. Aljinovic, L. A. Teot et al., "Methylmalonic acidemia: a megamitochondrial disorder affecting the kidney," Pediatric Nephrology, vol. 29, no. 11, pp. 21392146, 2014.

[91] Y. Wilnai, G. M. Enns, A. K. Niemi, J. Higgins, and H. Vogel, "Abnormal hepatocellular mitochondria in methylmalonic acidemia," Ultrastructural Pathology, vol. 38, no. 5, pp. 309314, 2014.

[92] J. Kim, C. Gherasim, and R. Banerjee, "Decyanation of vitamin B12 by a trafficking chaperone," Proceedings of the National Academy of Sciences, vol. 105, no. 38, pp. 1455114554, 2008.

[93] J. Kim, L. Hannibal, C. Gherasim, D. W. Jacobsen, and R. Banerjee, "A human vitamin B12 trafficking protein uses glutathione transferase activity for processing alkylcobalamins," Journal of Biological Chemistry, vol. 284, no. 48, pp. 33418-33424, 2009.

[94] Z. Li, C. Gherasim, N. A. Lesniak, and R. Banerjee, "Glutathione-dependent one-electron transfer reactions catalyzed by a $\mathrm{B}_{12}$ trafficking protein," Journal of Biological Chemistry, vol. 289, no. 23, pp. 16487-16497, 2014.
[95] Z. Li, A. Shanmuganathan, M. Ruetz et al., "Coordination chemistry controls the thiol oxidase activity of the $\mathrm{B}_{12}$-trafficking protein CblC," Journal of Biological Chemistry, vol. 292, no. 23, pp. 9733-9744, 2017.

[96] J. P. Lerner-Ellis, J. C. Tirone, P. D. Pawelek et al., "Identification of the gene responsible for methylmalonic aciduria and homocystinuria, cblC type," Nature Genetics, vol. 38, no. 1, pp. 93-100, 2005.

[97] J. D. Weisfeld-Adams, E. A. McCourt, G. A. Diaz, and S. C. Oliver, "Ocular disease in the cobalamin C defect: a review of the literature and a suggested framework for clinical surveillance," Molecular Genetics and Metabolism, vol. 114, no. 4, pp. 537-546, 2015.

[98] E. Richard, A. Jorge-Finnigan, J. Garcia-Villoria et al., "Genetic and cellular studies of oxidative stress in methylmalonic aciduria (MMA) cobalamin deficiency type C (cblC) with homocystinuria (MMACHC)," Human Mutation, vol. 30, no. 11, pp. 1558-1566, 2009.

[99] A. Jorge-Finnigan, A. Gamez, B. Perez, M. Ugarte, and E. Richard, "Different altered pattern expression of genes related to apoptosis in isolated methylmalonic aciduria cblB type and combined with homocystinuria cblC type," Biochimica et Biophysica Acta (BBA) - Molecular Basis of Disease, vol. 1802, no. 11, pp. 959-967, 2010.

[100] L. Hannibal, P. M. DiBello, M. Yu et al., “The MMACHC proteome: hallmarks of functional cobalamin deficiency in humans," Molecular Genetics and Metabolism, vol. 103, no. 3, pp. 226-239, 2011.

[101] L. Hannibal, P. M. DiBello, and D. W. Jacobsen, "Proteomics of vitamin B12 processing," Clinical Chemistry and Laboratory Medicine, vol. 51, no. 3, pp. 477-488, 2013.

[102] M. Caterino, A. Pastore, M. G. Strozziero et al., "The proteome of cblC defect: in vivo elucidation of altered cellular pathways in humans," Journal of Inherited Metabolic Disease, vol. 38, no. 5, pp. 969-979, 2015.

[103] C. Gherasim, M. Ruetz, Z. Li, S. Hudolin, and R. Banerjee, "Pathogenic mutations differentially affect the catalytic activities of the human B12-processing chaperone $\mathrm{CblC}$ and increase futile redox cycling," Journal of Biological Chemistry, vol. 290, no. 18, pp. 11393-11402, 2015.

[104] S. Goodman and F. Frerman, "Organic acidemias due to defects in lysine oxidation: 2-ketoadipic acidemia and glutaric acidemia," in The Metabolic and Molecular Basis of Inherited Disease, C. R. Scriver, A. L. Beaudet, W. S. Sly, and D. Valle, Eds., pp. 2195-2204, McGraw-Hill Inc, New York, 2001.

[105] S. Kölker, S. F. Garbade, C. R. Greenberg et al., "Natural history, outcome, and treatment efficacy in children and adults with glutaryl-CoA dehydrogenase deficiency," Pediatric Research, vol. 59, no. 6, pp. 840-847, 2006.

[106] K. A. Strauss, E. G. Puffenberger, D. L. Robinson, and D. H. Morton, "Type I glutaric aciduria, part 1: natural history of 77 patients," American Journal of Medical Genetics, vol. 121C, no. 1, pp. 38-52, 2003.

[107] A. U. Amaral, C. Cecatto, B. Seminotti et al., "Experimental evidence that bioenergetics disruption is not mainly involved in the brain injury of glutaryl-CoA dehydrogenase deficient mice submitted to lysine overload," Brain Research, vol. 1620, pp. 116-129, 2015.

[108] S. Kolker, S. W. Sauer, G. F. Hoffmann, I. Muller, M. A. Morath, and J. G. Okun, "Pathogenesis of CNS involvement in disorders of amino and organic acid metabolism," 
Journal of Inherited Metabolic Disease, vol. 31, no. 2, pp. 194-204, 2008.

[109] A. Latini, G. C. Ferreira, K. Scussiato et al., "Induction of oxidative stress by chronic and acute glutaric acid administration to rats," Cellular and Molecular Neurobiology, vol. 27, no. 4, pp. 423-438, 2007.

[110] D. M. Koeller, M. Woontner, L. S. Crnic et al., "Biochemical, pathologic and behavioral analysis of a mouse model of glutaric acidemia type I," Human Molecular Genetics, vol. 11, no. 4, pp. 347-357, 2002.

[111] B. Seminotti, R. T. Ribeiro, A. U. Amaral et al., "Acute lysine overload provokes protein oxidative damage and reduction of antioxidant defenses in the brain of infant glutaryl-CoA dehydrogenase deficient mice: a role for oxidative stress in GA I neuropathology," Journal of the Neurological Sciences, vol. 344, no. 1-2, pp. 105-113, 2014.

[112] W. J. Zinnanti, J. Lazovic, E. B. Wolpert et al., "A dietinduced mouse model for glutaric aciduria type I," Brain, vol. 129, no. 4, pp. 899-910, 2006.

[113] W. J. Zinnanti, J. Lazovic, C. Housman et al., "Mechanism of age-dependent susceptibility and novel treatment strategy in glutaric acidemia type I," Journal of Clinical Investigation, vol. 117, no. 11, pp. 3258-3270, 2007.

[114] C. Perez-Cerda, J. García-Villoria, R. Ofman et al., "2Methyl-3-hydroxybutyryl-CoA dehydrogenase (MHBD) deficiency: an X-linked inborn error of isoleucine metabolism that may mimic a mitochondrial disease," Pediatric Research, vol. 58, no. 3, pp. 488-491, 2005.

[115] W. Sperl, P. Ješina, J. Zeman et al., "Deficiency of mitochondrial ATP synthase of nuclear genetic origin," Neuromuscular Disorders, vol. 16, no. 12, pp. 821-829, 2006.

[116] C. G. da Silva, C. A. J. Ribeiro, G. Leipnitz et al., "Inhibition of cytochrome $\mathrm{c}$ oxidase activity in rat cerebral cortex and human skeletal muscle by D-2-hydroxyglutaric acid in vitro," Biochimica et Biophysica Acta (BBA) - Molecular Basis of Disease, vol. 1586, no. 1, pp. 81-91, 2002.

[117] D. Zafeiriou, P. Augoustides-Savvopoulou, D. Haas et al., "Ethylmalonic encephalopathy: clinical and biochemical observations," Neuropediatrics, vol. 38, no. 2, pp. 78-82, 2007.

[118] S. B. Wortmann, R. J. T. Rodenburg, A. Jonckheere et al., "Biochemical and genetic analysis of 3-methylglutaconic aciduria type IV: a diagnostic strategy," Brain, vol. 132, no. 1, pp. 136-146, 2009.

[119] L. Zandberg, H. C. van Dyk, F. H. van der Westhuizen, and A. A. van Dijk, "A 3-methylcrotonyl-CoA carboxylase deficient human skin fibroblast transcriptome reveals underlying mitochondrial dysfunction and oxidative stress," The International Journal of Biochemistry \& Cell Biology, vol. 78, pp. 116-129, 2016.

[120] Â. Zanatta, A. P. Moura, A. M. Tonin et al., "Neurochemical evidence that the metabolites accumulating in 3methylcrotonyl-CoA carboxylase deficiency induce oxidative damage in cerebral cortex of young rats," Cellular and Molecular Neurobiology, vol. 33, no. 1, pp. 137-146, 2013.

[121] M. R. Hayden and S. C. Tyagi, "Homocysteine and reactive oxygen species in metabolic syndrome, type 2 diabetes mellitus, and atheroscleropathy: the pleiotropic effects of folate supplementation," Nutrition Journal, vol. 3, no. 1, 2004.

[122] C. G. Zou and R. Banerjee, "Homocysteine and redox signaling," Antioxidants \& Redox Signaling, vol. 7, no. 5-6, pp. 547559, 2005.
[123] D. W. Jacobsen, "Hyperhomocysteinemia and oxidative stress: time for a reality check?," Arteriosclerosis, Thrombosis, and Vascular Biology, vol. 20, no. 5, pp. 1182-1184, 2000.

[124] H. J. Blom, "Consequences of homocysteine export and oxidation in the vascular system," Seminars in Thrombosis and Hemostasis, vol. Volume 26, no. Number 03, pp. 227-232, 2000.

[125] N. Tyagi, K. C. Sedoris, M. Steed, A. V. Ovechkin, K. S. Moshal, and S. C. Tyagi, "Mechanisms of homocysteineinduced oxidative stress," American Journal of PhysiologyHeart and Circulatory Physiology, vol. 289, no. 6, pp. H2649-H2656, 2005.

[126] F. Bonetti, G. Brombo, and G. Zuliani, "The relationship between hyperhomocysteinemia and neurodegeneration," Neurodegenerative Disease Management, vol. 6, no. 2, pp. 133-145, 2016.

[127] S. H. Mudd, H. L. Levy, and J. P. Kraus, "Disorders of transsulfuration," in The Online Metabolic and Molecular Bases of Inherited Diseases, K. Kinzler, A. Ballabio, S. E. Antonarakis, A. L. Beaudet, B. Vogelstein, and D. Valle, Eds., McGrall-Hill, New York, 2011.

[128] M. Schiff and H. J. Blom, "Treatment of inherited homocystinurias," Neuropediatrics, vol. 43, no. 6, pp. 295-304, 2012.

[129] J. L. Faverzani, T. G. Hammerschmidt, A. Sitta, M. Deon, M. Wajner, and C. R. Vargas, "Oxidative stress in homocystinuria due to cystathionine $\beta$-synthase deficiency: findings in patients and in animal models," Cellular and Molecular Neurobiology, vol. 37, no. 8, pp. 1477-1485, 2017.

[130] C. S. Vanzin, G. B. Biancini, A. Sitta et al., "Experimental evidence of oxidative stress in plasma of homocystinuric patients: a possible role for homocysteine," Molecular Genetics and Metabolism, vol. 104, no. 1-2, pp. 112-117, 2011.

[131] C. S. Vanzin, V. Manfredini, A. E. Marinho et al., "Homocysteine contribution to DNA damage in cystathionine $\beta$-synthase-deficient patients," Gene, vol. 539, no. 2, pp. 270-274, 2014.

[132] C. S. Vanzin, C. P. Mescka, B. Donida et al., "Lipid, oxidative and inflammatory profile and alterations in the enzymes paraoxonase and butyrylcholinesterase in plasma of patients with homocystinuria due CBS deficiency: the vitamin B12 and folic acid importance," Cellular and Molecular Neurobiology, vol. 35, no. 6, pp. 899-911, 2015.

[133] K. Robert, J. Nehme, E. Bourdon et al., "Cystathionine $\beta$ synthase deficiency promotes oxidative stress, fibrosis, and steatosis in mice liver," Gastroenterology, vol. 128, no. 5, pp. 1405-1415, 2005.

[134] E. L. Streck, P. S. Vieira, C. M. Wannmacher, C. S. DutraFilho, M. Wajner, and A. T. Wyse, "In vitro effect of homocysteine on some parameters of oxidative stress in rat hippocampus," Metabolic Brain Disease, vol. 18, no. 2, pp. 147-154, 2003.

[135] C. Matté, F. M. Stefanello, V. Mackedanz et al., "Homocysteine induces oxidative stress, inflammatory infiltration, fibrosis and reduces glycogen/glycoprotein content in liver of rats," International Journal of Developmental Neuroscience, vol. 27, no. 4, pp. 337-344, 2009.

[136] D. Watkins and D. S. Rosenblatt, "Inherited disorders of folate and cobalamin transport amd metabolism," in The Metabolic and Molecular Bases of Inherited Diseases, C. R. Scriver, A. L. Beaudet, W. Sly, and D. Valle, Eds., pp. 38973933, McGrall-Hill, New York, 2011. 
[137] R. Surtees, "Demyelination and inborn errors of the single carbon transfer pathway," European Journal of Pediatrics, vol. 157, no. S2, pp. S118-S121, 1998.

[138] A. Martinez-Pizarro, L. R. Desviat, M. Ugarte, B. Perez, and E. Richard, "Endoplasmic reticulum stress and autophagy in homocystinuria patients with remethylation defects," PLoS One, vol. 11, no. 3, article e0150357, 2016.

[139] S. I. Dikalov and D. G. Harrison, "Methods for detection of mitochondrial and cellular reactive oxygen species," Antioxidants \& Redox Signaling, vol. 20, no. 2, pp. 372-382, 2014.

[140] X. Zhang and F. Gao, "Imaging mitochondrial reactive oxygen species with fluorescent probes: current applications and challenges," Free Radical Research, vol. 49, no. 4, pp. 374-382, 2015.

[141] B. Kalyanaraman, M. Hardy, R. Podsiadly, G. Cheng, and J. Zielonka, "Recent developments in detection of superoxide radical anion and hydrogen peroxide: opportunities, challenges, and implications in redox signaling," Archives of Biochemistry and Biophysics, vol. 617, pp. 38-47, 2017.

[142] K. Debowska, D. Debski, M. Hardy et al., "Toward selective detection of reactive oxygen and nitrogen species with the use of fluorogenic probes - limitations, progress, and perspectives," Pharmacological Reports, vol. 67, no. 4, pp. 756-764, 2015.

[143] W. A. Pryor and S. S. Godber, "Noninvasive measures of oxidative stress status in humans," Free Radical Biology and Medicine, vol. 10, no. 3-4, pp. 177-184, 1991.

[144] A. C. Ribou, "Synthetic sensors for reactive oxygen species detection and quantification: a critical review of current methods," Antioxidants \& Redox Signaling, vol. 25, no. 9, pp. 520-533, 2016.

[145] M. Wrona and P. Wardman, "Properties of the radical intermediate obtained on oxidation of $2^{\prime}, 7^{\prime}$-dichlorodihydrofluorescein, a probe for oxidative stress," Free Radical Biology and Medicine, vol. 41, no. 4, pp. 657-667, 2006.

[146] C. Rota, C. F. Chignell, and R. P. Mason, "Evidence for free radical formation during the oxidation of $2^{\prime}-7^{\prime}$-dichlorofluorescin to the fluorescent dye $2^{\prime}-7^{\prime}$-dichlorofluorescein by horseradish peroxidase:possible implications for oxidative stress measurements," Free Radical Biology and Medicine, vol. 27, no. 7-8, pp. 873-881, 1999.

[147] L. F. F. Royes, P. Gabbi, L. R. Ribeiro et al., "A neuronal disruption in redox homeostasis elicited by ammonia alters the glycine/glutamate (GABA) cycle and contributes to MMA-induced excitability," Amino Acids, vol. 48, no. 6, pp. 1373-1389, 2016.

[148] H. Zhao, J. Joseph, H. M. Fales et al., "Detection and characterization of the product of hydroethidine and intracellular superoxide by HPLC and limitations of fluorescence," Proceedings of the National Academy of Sciences, vol. 102, no. 16, pp. 5727-5732, 2005.

[149] K. M. Robinson, M. S. Janes, M. Pehar et al., "Selective fluorescent imaging of superoxide in vivo using ethidium-based probes," Proceedings of the National Academy of Sciences, vol. 103, no. 41, pp. 15038-15043, 2006.

[150] J. Zielonka, M. Hardy, and B. Kalyanaraman, "HPLC study of oxidation products of hydroethidine in chemical and biological systems: ramifications in superoxide measurements," Free Radical Biology and Medicine, vol. 46, no. 3, pp. 329338, 2009.
[151] A. Logan, H. M. Cochemé, P. B. L. Pun et al., "Using exomarkers to assess mitochondrial reactive species in vivo," Biochimica et Biophysica Acta (BBA) - General Subjects, vol. 1840, no. 2, pp. 923-930, 2014.

[152] A. Logan, I. G. Shabalina, T. A. Prime et al., "In vivo levels of mitochondrial hydrogen peroxide increase with age in mtDNA mutator mice," Aging Cell, vol. 13, no. 4, pp. 765768, 2014.

[153] B. Merinero, C. Perez-Cerda, L. R. Desviat et al., "Mitochondrial organic acidurias. Part I: biochemical and molecular basis," in Mitochondrial Pathophysiology, F. Palau and S. Cadenas, Eds., pp. 145-171, Research Signpost, Kerala (India), 2011.

[154] H. J. Forman, K. J. A. Davies, and F. Ursini, "How do nutritional antioxidants really work: nucleophilic tone and para-hormesis versus free radical scavenging in vivo," Free Radical Biology and Medicine, vol. 66, pp. 24-35, 2014.

[155] H. H. H. W. Schmidt, R. Stocker, C. Vollbracht et al., "Antioxidants in translational medicine," Antioxidants \& Redox Signaling, vol. 23, no. 14, pp. 1130-1143, 2015.

[156] J. D. Hayes and A. T. Dinkova-Kostova, "The Nrf2 regulatory network provides an interface between redox and intermediary metabolism," Trends in Biochemical Sciences, vol. 39, no. 4, pp. 199-218, 2014.

[157] A. Brunet, L. B. Sweeney, J. F. Sturgill et al., "Stress-dependent regulation of FOXO transcription factors by the SIRT1 deacetylase," Science, vol. 303, no. 5666, pp. 2011-2015, 2004.

[158] S. Nemoto, M. M. Fergusson, and T. Finkel, "SIRT1 functionally interacts with the metabolic regulator and transcriptional coactivator PGC-1 $\alpha$," Journal of Biological Chemistry, vol. 280, no. 16, pp. 16456-16460, 2005.

[159] L. F. Pettenuzzo, Patrícia F. Schuck, A. T. S. Wyse et al., "Ascorbic acid prevents water maze behavioral deficits caused by early postnatal methylmalonic acid administration in the rat," Brain Research, vol. 976, no. 2, pp. 234-242, 2003.

[160] L. Pettenuzzo, P. F. Schuck, F. Fontella et al., "Ascorbic acid prevents cognitive deficits caused by chronic administration of propionic acid to rats in the water maze," Pharmacology Biochemistry and Behavior, vol. 73, no. 3, pp. 623-629, 2002.

[161] C. A. Ribeiro, Â. M. Sgaravatti, R. B. Rosa et al., "Inhibition of brain energy metabolism by the branched-chain amino acids accumulating in maple syrup urine disease," Neurochemical Research, vol. 33, no. 1, pp. 114-124, 2008.

[162] F. S. Rodrigues, M. A. Souza, D. V. Magni et al., "N-Acetylcysteine prevents spatial memory impairment induced by chronic early postnatal glutaric acid and lipopolysaccharide in rat pups," PLoS One, vol. 8, no. 10, article e78332, 2013.

[163] G. S. Ribas, V. Manfredini, J. F. de Mari et al., "Reduction of lipid and protein damage in patients with disorders of propionate metabolism under treatment: a possible protective role of L-carnitine supplementation," International Journal of Developmental Neuroscience, vol. 28, no. 2, pp. 127-132, 2010.

[164] I. Gulcin, "Antioxidant and antiradical activities of L-carnitine," Life Sciences, vol. 78, no. 8, pp. 803-811, 2006.

[165] G. Guerreiro, C. P. Mescka, A. Sitta et al., "Urinary biomarkers of oxidative damage in maple syrup urine disease: the L-carnitine role," International Journal of Developmental Neuroscience, vol. 42, pp. 10-14, 2015.

[166] S. Pinar-Sueiro, R. Martinez-Fernandez, S. Lage-Medina, L. Aldamiz-Echevarria, and E. Vecino, "Optic neuropathy 
in methylmalonic acidemia: the role of neuroprotection," Journal of Inherited Metabolic Disease, vol. 33, no. S3, pp. 199-203, 2010.

[167] A. J. Guenzel, S. E. Hofherr, M. Hillestad et al., "Generation of a hypomorphic model of propionic acidemia amenable to gene therapy testing," Molecular Therapy, vol. 21, no. 7, pp. 1316-1323, 2013.

[168] M. R. de Oliveira, S. F. Nabavi, A. Manayi, M. Daglia, Z. Hajheydari, and S. M. Nabavi, "Resveratrol and the mitochondria: from triggering the intrinsic apoptotic pathway to inducing mitochondrial biogenesis, a mechanistic view," Biochimica et Biophysica Acta (BBA) - General Subjects, vol. 1860, no. 4, pp. 727-745, 2016.

[169] A. Lopes Costa, C. Le Bachelier, L. Mathieu et al., "Beneficial effects of resveratrol on respiratory chain defects in patients' fibroblasts involve estrogen receptor and estrogen-related receptor alpha signaling," Human Molecular Genetics, vol. 23, no. 8, pp. 2106-2119, 2014.

[170] A. W. El-Hattab, A. M. Zarante, M. Almannai, and F. Scaglia, "Therapies for mitochondrial diseases and current clinical trials," Molecular Genetics and Metabolism, vol. 122, no. 3, pp. 1-9, 2017.

[171] K. N. Maclean, H. Jiang, S. Aivazidis et al., "Taurine treatment prevents derangement of the hepatic $\gamma$-glutamyl cycle and methylglyoxal metabolism in a mouse model of classical homocystinuria: regulatory crosstalk between thiol and sulfinic acid metabolism," The FASEB Journal, article fj.201700586R, 2018.

[172] O. Firuzi, R. Miri, M. Tavakkoli, and L. Saso, "Antioxidant therapy: current status and future prospects," Current Medicinal Chemistry, vol. 18, no. 25, pp. 3871-3888, 2011.

[173] P. Palozza, S. Serini, A. Torsello et al., "Regulation of cell cycle progression and apoptosis by beta-carotene in undifferentiated and differentiated HL-60 leukemia cells: possible involvement of a redox mechanism," International Journal of Cancer, vol. 97, no. 5, pp. 593-600, 2002.

[174] S. L. Yeh, H. M. Wang, P. Y. Chen, and T. C. Wu, "Interactions of $\beta$-carotene and flavonoids on the secretion of pro-inflammatory mediators in an in vitro system," Chemico-Biological Interactions, vol. 179, no. 2-3, pp. 386393, 2009.

[175] J. Lin, N. R. Cook, C. Albert et al., "Vitamins C and E and beta carotene supplementation and cancer risk: a randomized controlled trial," Journal of the National Cancer Institute, vol. 101, no. 1, pp. 14-23, 2009.

[176] G. Bjelakovic, D. Nikolova, L. L. Gluud, R. G. Simonetti, and C. Gluud, "Mortality in randomized trials of antioxidant supplements for primary and secondary prevention: systematic review and meta-analysis," JAMA, vol. 297 , no. 8 , pp. $842-$ 857, 2007.

[177] G. Bjelakovic, D. Nikolova, L. L. Gluud, R. G. Simonetti, and C. Gluud, "Antioxidant supplements for prevention of mortality in healthy participants and patients with various diseases," Cochrane Database of Systematic Reviews, no. 3, article CD007176, 2012.

[178] K. R. Martin and J. C. Barrett, "Reactive oxygen species as double-edged swords in cellular processes: low-dose cell signaling versus high-dose toxicity," Human \& Experimental Toxicology, vol. 21, no. 2, pp. 71-75, 2002.
[179] L. I. Leichert and T. P. Dick, "Incidence and physiological relevance of protein thiol switches," Biological Chemistry, vol. 396, no. 5, pp. 389-399, 2015.

[180] Y. Fujikawa, L. P. Roma, M. C. Sobotta et al., "Mouse redox histology using genetically encoded probes," Science Signaling, vol. 9, no. 419, p. rs1, 2016. 


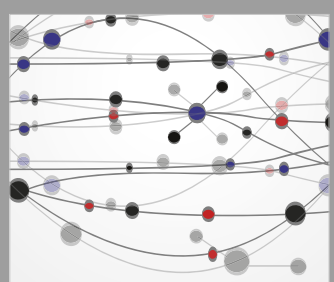

The Scientific World Journal
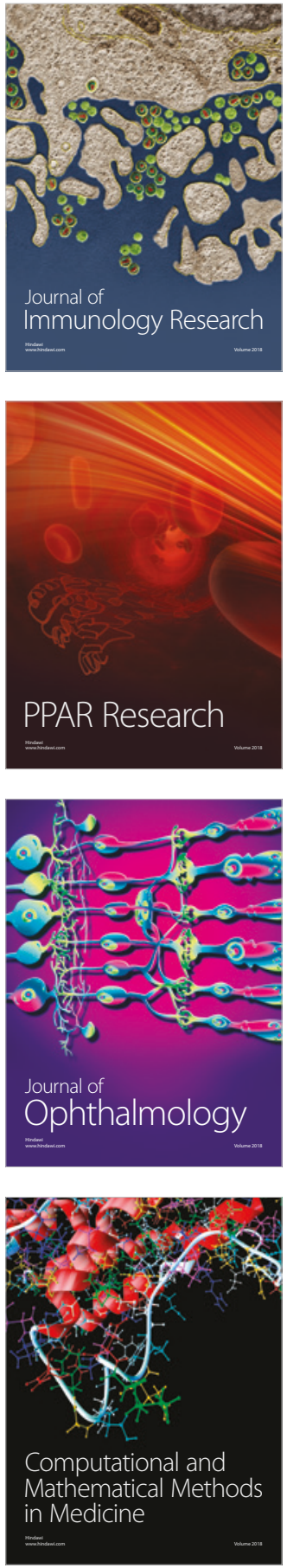

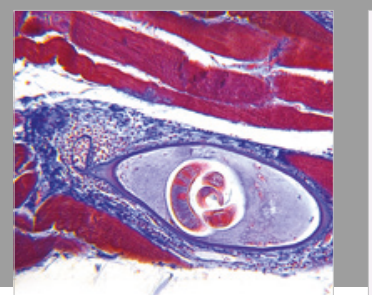

Gastroenterology Research and Practice

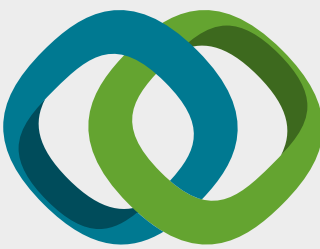

\section{Hindawi}

Submit your manuscripts at

www.hindawi.com
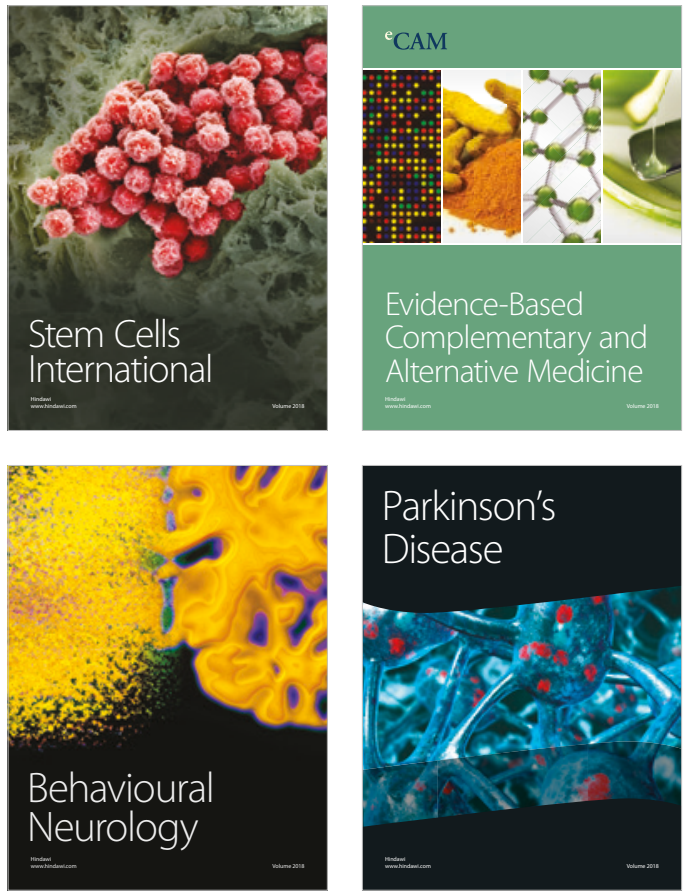

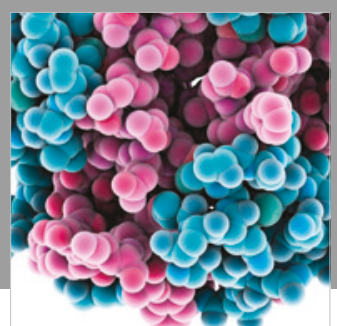

ournal of

Diabetes Research

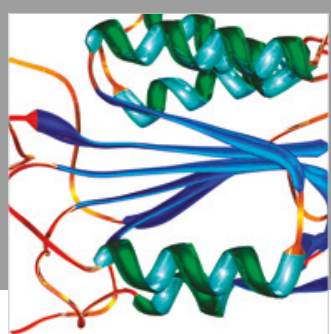

Disease Markers
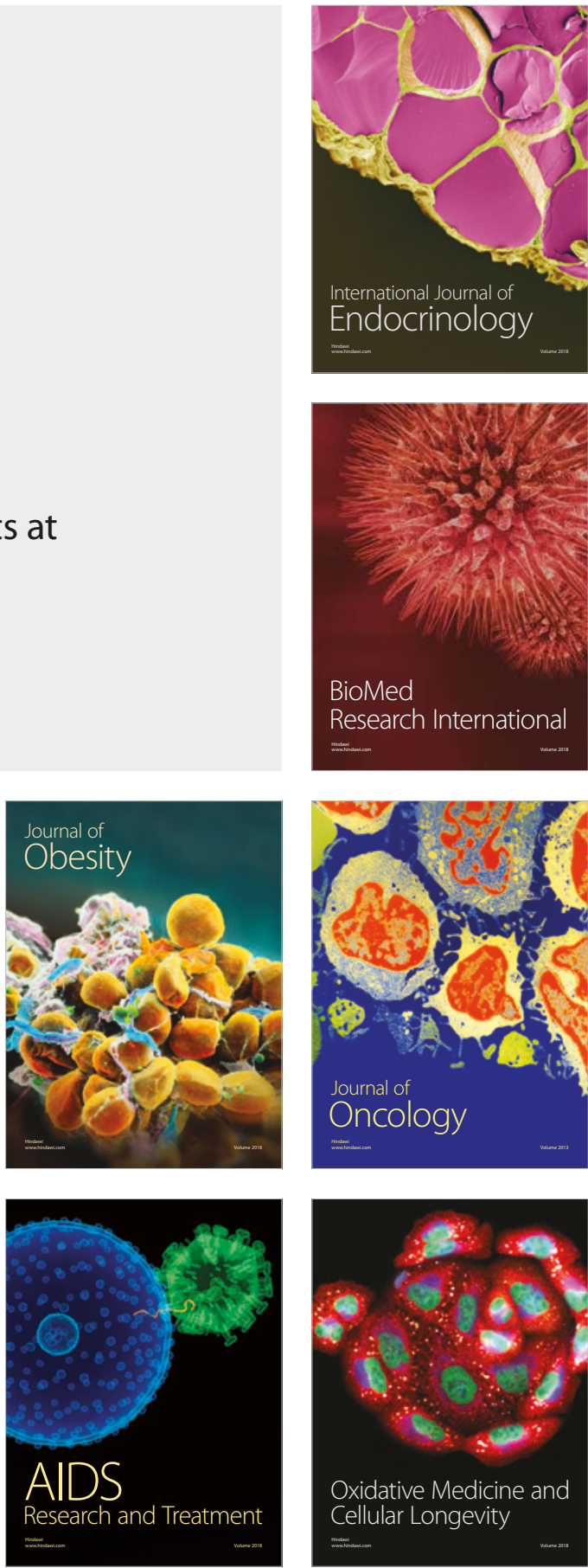